\title{
Pre-participation cardiovascular evaluation for athletic participants to prevent sudden death: Position paper from the EHRA and the EACPR, branches of the ESC. Endorsed by APHRS,
} HRS, and SOLAECE

\begin{abstract}
Lluís Mont ${ }^{1}$, Antonio Pelliccia ${ }^{2}$, Sanjay Sharma ${ }^{3}$, Alessandro Biffi ${ }^{2}$, Mats Borjesson ${ }^{4}$, Josep Brugada Terradellas ${ }^{1}$, François Carré ${ }^{5}$, Eduard Guasch ${ }^{1}$, Hein Heidbuchel ${ }^{6}$, André La Gerche ${ }^{7}$, Rachel Lampert ${ }^{8}$, William McKenna ${ }^{9}$, Michail Papadakis ${ }^{3}$, Silvia G. Priori ${ }^{10}$, Mauricio Scanavacca ${ }^{11}$, Paul Thompson ${ }^{12}$, Christian Sticherling ${ }^{13}$, Sami Viskin ${ }^{14}$, Mathew Wilson ${ }^{15}$, and Domenico Corrado ${ }^{16}$
\end{abstract}

Reviewers: Gregory Y.H. Lip, (Review Coordinator) ${ }^{17}$, Bulent Gorenek ${ }^{18}$, Carina Blomström Lundqvist ${ }^{19}$, Bela Merkely ${ }^{20}$, Gerhard Hindricks ${ }^{21}$, Antonio Hernández-Madrid ${ }^{22}$, Deirdre Lane ${ }^{17}$, Guiseppe Boriani ${ }^{23}$, Calambur Narasimhan ${ }^{24}$, Manlio F. Marquez ${ }^{25}$, David Haines ${ }^{26}$, Judith Mackall ${ }^{27}$, Pedro Manuel Marques-Vidal ${ }^{28}$, Ugo Corra ${ }^{29}$, Martin Halle ${ }^{30}$, Monica Tiberi ${ }^{31}$, Josef Niebauer ${ }^{32}$, and Massimo Piepoli33

\footnotetext{
${ }^{1}$ Hospital Clinic, Universitat de Barcelona, Barcelona, Spain; ${ }^{2}$ Institute of Sport Medicine and Science, Rome, Italy; ${ }^{3}$ St George's Hospital NHS Trust, London, UK; ${ }^{4}$ Inst of Neuroscience and Physiology and Food, Nutrition and Sport Science and Östra University Hospital, Göteborg, Sweden; ${ }^{5}$ Hopital Pontchaillou, Rennes, France; ${ }^{6}$ Heart Center Hasselt, Hasselt, Belgium; ${ }^{7}$ Baker IDI Heart and Diabetes Institute, Melbourne, Australia; ${ }^{8}$ Yale University School of Medicine, New Haven, CT, USA; ${ }^{9}$ The Heart Hospital, University College London, London, UK; ${ }^{10}$ IRCCS Fondazione Salvatore Maugeri, Pavia, Italy; ${ }^{11}$ Instituto do Coração (InCor) do Hospital das Clinicas da Faculdade de Medicina da Universidade de São Paulo, São Paulo, Brazil; ${ }^{12}$ Hartford Hospital, Hartford, CT, USA; ${ }^{13}$ University Hospital Basel, Basel, Switzerland; ${ }^{14}$ Tel Aviv Medical Center, Tel Aviv, Israel; ${ }^{15}$ Aspetar - Sports Medicine Department, Doha, Qatar; ${ }^{16}$ University of Padova, Padova, Italy; ${ }^{17}$ University of Birmingham, Birmingham, UK; ${ }^{18}$ Eskisehir Osmangazi University, Eskisehir, Turkey; ${ }^{19}$ University Hospital in Uppsala, Uppsala, Sweden; ${ }^{20}$ Semmelweis University, Heart and Vascular Center, Budapest, Hungary; ${ }^{21}$ University of Leipzig, Heartcenter, Leipzig, Germany; ${ }^{22}$ Unidad De Arritmias, Servicio De Cardiologia, Hospital Universitario Ramón y Cajal, Madrid, Spain; ${ }^{23}$ University of Modena and Reggio Emilia, Modena, Italy; ${ }^{24}$ Cardiology Department, Care Hospital, Hyderabad, India; ${ }^{25}$ Departamento de Electrocardiología, Instituto Nacional de Cardiologia Ignacio Chavez, Tlalpan, Mexico; ${ }^{26}$ Department of Cardiovascular Medicine, Beaumont Health System, Royal Oak, MI, USA; ${ }^{27}$ University Hospitals Case Medical Center, Cleveland, OH, USA; ${ }^{28}$ University Hospital of Lausanne, Lausanne, Switzerland; ${ }^{29}$ IRCCS Rehabilitation Medical Center, Cardiology Department, Salvatore Maugeri Foundation, Veruno, Italy; ${ }^{30}$ Prevention and Sports Medicine, Technical University Munich, Munich, Germany; ${ }^{31}$ Studio Medico Sportivo, Pesaro, Italy; ${ }^{32}$ Sports Medicine, Prevention \& Rehabilitation, Paracelsus Medical University, Salzburg, Austria; and ${ }^{33}$ Polichirurgico Hospital G. Da Saliceto, Romagna, Italy
}

Online publish-ahead-of-print 4 November 2016

\section{Table of contents}

Introduction ........................... 140

Incidence of sudden cardiac death in athletes .......... 140

Causes of sudden cardiac death in athletes ........... 141

Hypertrophic cardiomyopathy. . . . . . . . . . . . . . . 141

Arrhythmogenic right ventricular cardiomyopathy ....... 144

Coronary congenital abnormalities .................. 144

Ventricular pre-excitation ...................... 145

Left ventricle non-compaction .................. 146

Dilated cardiomyopathy ...................... 147

Valvulopathies ........................... 147
Inherited primary arrhythmia syndromes .......... 148

Long QT syndrome ................... 148

Short QT syndrome . . . . . . . . . . . . . . . 148

Catecholaminergic polymorphic ventricular tachycardia . 149

Brugada syndrome ..................... 150

Acquired cardiac abnormalities ................ 150

Coronary artery disease . . . . . . . . . . . . . . 150

Chagas disease ....................... 151

Myocarditis .......................... 152

Exercise as a promoter of cardiac injury . . . . . . 152

Role of screening techniques .................. 152

Role of electrocardiogram .................. 152 
Role of ECG exercise test .................... 154

Role of ambulatory ECG monitoring ............ 154

Role of imaging modalities in screening . . . . . . . . 155

Echocardiography ...................... 155

Cardiac magnetic resonance imaging . . . . . . . . 155

Computed Tomography [...] coronary angiography and cor-

onary artery calcium scoring . . . . . . . . . . 155

Existing screening programmes, rationale, and limitations . . . 156

Evidence supporting preparticipation evaluation programmes: impact on cardiovascular mortality .............. 156

Cost-effectiveness considerations . . . . . . . . . . . 157

Consensus statements ..................... 158

Aim and nature of the preparticipation evaluation...... 159

Protocol of the preparticipation evaluation .......... 159

Implementation of the preparticipation evaluation ...... 159

Future directions ........................ 159

References .............................. 159

\section{Abbreviation list}

$\begin{array}{ll}\text { AMI } & \text { acute myocardial infarction } \\ \text { ARVC } & \text { arrhythmogenic right ventricular cardiomyopathy } \\ \text { BrS } & \text { Brugada syndrome } \\ \text { CACS } & \text { coronary artery calcium score } \\ \text { CAD } & \text { coronary artery disease } \\ \text { ChD } & \text { Chagas heart disease } \\ \text { CMR } & \text { cardiac magnetic resonance } \\ \text { CPVT } & \text { catecholaminergic polymorphic ventricular tachycardia } \\ \text { CTCA } & \text { computed tomography coronary angiography } \\ \text { CV } & \text { cardiovascular } \\ \text { DCM } & \text { dilated cardiomyopathy } \\ \text { EAPCR } & \text { European Association for Cardiovascular Prevention } \\ & \text { and Rehabilitation } \\ \text { HCM } & \text { hypertrophic cardiomyopathy } \\ \text { LGE } & \text { late gadolinium enhancement } \\ \text { LQTS } & \text { long QT syndrome } \\ \text { LV/RV } & \text { left/right ventricle } \\ \text { LVH } & \text { left ventricle hypertrophy } \\ \text { NSVT } & \text { non-sustained ventricular tachycardia } \\ \text { PPE } & \text { preparticipation evaluation } \\ \text { PVC } & \text { premature ventricular contractions } \\ \text { SCA/ } & \text { sudden cardiac arrest/death } \\ \text { SCD } & \\ \text { TTE } & \text { transthoracic echocardiography } \\ \text { VF } & \text { ventricular fibrillation } \\ \text { VT } & \text { ventricular tachycardia } \\ & \end{array}$

\section{Introduction}

Sudden cardiac death (SCD) associated with athletic activity is a rare but devastating event. Victims are usually young and apparently healthy, and while many of these deaths remain unexplained, a substantial number of victims harbour an underlying and potentially detectable cardiovascular (CV) disease. ${ }^{1-4}$ The vast majority of these events are due to malignant tachyarrhythmias, usually ventricular fibrillation (VF) or ventricular tachycardia (VT) degenerating into ventricular fibrillation (VF), occurring in individuals with arrhythmogenic disorders (e.g. hypertrophic cardiomyopathy, arrhythmogenic cardiomyopathy, channelopathies). Intensive exercise training and competitive sport participation is a trigger that may favour insurgence of ominous ventricular tachyarrhythmias in predisposed individuals. ${ }^{5}$ Consequently, there is a great interest in early identification of at-risk individuals for whom appropriate treatment, followed or not by physical activity adjustment, may be implemented to minimize the risk of SCD. However, the role of pre-participation evaluation (PPE) in athletes as a feasible and efficient strategy to identify individuals at risk has remained controversial.

There are several points that have been debated in the recent years, including the actual incidence of acute cardiac events in the athlete's population, the diagnostic capability, as well as the specificity and sensitivity of the testing commonly in use to identify the cardiac conditions at-risk (particularly, but not only, ${ }^{6} 12$-lead ECG), the impact of the screening protocols on the clinical outcome, and finally the cost/efficacy considerations.

The target of this document is 'athletic participants', here defined as individuals of any age, either amateur or professional, who are engaged in regular exercise training, independent of the competitive status. We acknowledge that not uncommonly athletic participants, regardless of the level of achievement, have the inclination to exert physically up to their limits to improve performance.

The purpose of the present position paper promoted by EHRA and EACPR and endorsed by APHRS, HRS, and SOLAECE, is to review the scientific evidence regarding the appropriate diagnostic methods to identify the cardiac conditions at risk in the athletic population, discuss the role of the different tests in the context of PPE and, finally, offer the consensus opinions relative to the most efficient pre-participation screening to improve safe sport participation, according to the current scientific evidence and-if necessary - to update the last recommendations on this topic. ${ }^{7,8}$ Notably, consequences of PPE such as the most appropriate medical treatment for a certain condition or criteria to be used for disqualification from intensive exercise are discussed elsewhere. ${ }^{7,9-12}$

Our interest for safe sport participation is dictated by the awareness that exercise and sport activities are already a component of the lifestyle in a large subset of our societies, and are recommended by the current guidelines for maintaining $\mathrm{CV}$ health and preventing disease. $^{13}$

\section{Incidence of sudden cardiac death in athletes}

Sports-related SCD is usually defined as sudden and unexpected death occurring during, or shortly after, exercise (with varying time intervals up to $24 \mathrm{~h}$ used by different investigators), if witnessed by a bystander and/or happening in an individual who was otherwise known to be healthy. The true incidence of SCD in athletes remains uncertain. Nonetheless, its answer is important to gauge the effect of measures to reduce it, i.e. PPE.

A good overview of existing datasets was recently provided by Harmon et al. ${ }^{14}$ The problem in determining the magnitude of the incidence of SCD lies in multiple factors. Calculation requires exact estimates of both the numerator (how many athletes died suddenly?) and the denominator (how large is the population in which these 
cases developed?). Indeed, in an era with more rapid response systems and on-site availability of automatic external defibrillators, not only fatalities need to be counted (i.e. sudden death), but also survivors of sudden cardiac arrest (SCA).

Very few studies are rigorous in the estimates of both the numerator and denominator. Since age has an important impact on the incidence of sports-related SCDs, it is also important to clearly define the age groups when describing estimates. Finally, from a public health perspective, both sudden deaths of athletes during sports and during rest should be recorded.

As an overall estimate, 1 to 2 out of 100000 athletes between the age of 12 and 35 years die suddenly each year. ${ }^{2,3,5,15-17}$ The most robust data come from Italian studies, relying on mandatory reporting of SCDs and on good population demographics, since Italian law mandates regular medical evaluation in all participants of organized sports. ${ }^{5,18}$ Retrospective US cohort studies in college athletes, ${ }^{17}$ a prospective observational study in high-school athletes, ${ }^{19}$ and studies in active US military recruits ${ }^{20}$ reported similar figures.

On average, the Italian experience suggests that there is a three times higher incidence of SCDs in athletes (estimated at 2.3 per 100000 individuals) than in non-athletes (0.9 per 100000 individuals). ${ }^{5}$ Recently, however, Danish investigators could not reproduce these results and found a reduced SCD in athletes. ${ }^{21}$ Sport is not considered in itself the cause of the enhanced mortality; rather, it acts as a trigger of life-threatening ventricular arrhythmias and SCA in predisposed individuals. The incidence of SCD is similar in competitive and recreational athletes. ${ }^{16}$

The incidence is highly gender-dependent, with rates in females being 2-25 times lower than in men, the reasons of which are not well understood. $2,5,14,17-19$ The incidence also steeply increases with age, being about 5-10 times higher in those over 35 years old compared to younger athletes. ${ }^{2,14,17-19}$ Italian and US data did not demonstrate a clear correlation between type of sports and SCD/ SCA incidence. ${ }^{2,14,17-19}$ On the other hand, however, data from highschool and college athletes in the US point to specific athlete populations with increased incidence: particularly black athletes (5.5/ $100000)$ and male basketball players ( $>10 / 100000)$ seem to have a higher risk. ${ }^{2,14}$ Again, the causes for these associations are not clear.

Efforts should be made to set up better registration of sportsrelated SCDs. The deficiencies of relying on media reports or insurance claims databases have been shown. ${ }^{2,14,22}$ Good registration requires nationwide reporting of death, including description of the circumstances of death in relation to exercise, and of the habitual exercise level of the deceased. Ideally, this should be complemented with a uniform evaluation by autopsy and post-mortem genotyping. ${ }^{22}$

\section{Causes of sudden cardiac death in athletes}

We will review the main characteristics of the most frequent and emergent cardiovascular conditions involved in SCD. It should be noted, though, that the most common conditions are variable depending on factors such as age and geographical localization of the studied population. A brief overview of these conditions are summarized in Table 1.

\section{Hypertrophic cardiomyopathy}

Hypertrophic cardiomyopathy (HCM) is defined by left ventricular hypertrophy $(\mathrm{LVH})$ that is not solely explained by abnormal loading conditions. Most recent guidelines do not make a priori assumptions about aetiology or pathology. Under this definition, mutations in genes encoding sarcomeric proteins account for $60 \%$ of young and middle-aged cases. ${ }^{9} \mathrm{HCM}$ is one of the most common conditions in young athletes. The prevalence of phenotypically overt HCM has been estimated at 1:500 in European, American, Asian, and African populations. ${ }^{9,25,26}$ Familial evaluation suggests that most individuals who carry disease-causing mutations will have incomplete penetrance, not uncommonly presenting with an abnormal electrocardiogram but without fulfilling conventional imaging diagnostic criteria. ${ }^{27}$ Therefore, the prevalence of HCM-causing mutations is likely higher, in the range of $1: 200 .^{26}$

Patients may remain asymptomatic over a long course of their disease. Clinical manifestations may include heart failure and atrial fibrillation, whereas SCD can be the first disease manifestation in others. ${ }^{9}$ A recently published clinical risk prediction model provides a 5 year sudden death risk estimation upon which preventive strategies could be established. ${ }^{28}$

Exercise and sport in HCM patients. In most series, primarily from the U.S., HCM is the most common cause of SCD in young individuals engaged in competitive sports. ${ }^{14,23}$ Strenuous exercise exposes individuals with HCM to an increased vulnerability because of potential triggers [e.g. ischaemia, left ventricle (LV) outflow tract obstruction, abnormal vascular responses, atrial fibrillation] for hypotension and ventricular arrhythmia.

Diagnosis. Diagnosis of HCM requires imaging testing (2-dimensional echocardiography or magnetic resonance imaging); an otherwise unexplained LVH, with wall thickness $\geq 13 \mathrm{~mm}$ in $\mathrm{fe}$ males, $\geq 15 \mathrm{~mm}$ in males, or $>$ three standard deviations from the body surface area-adjusted average is considered diagnostic. ${ }^{9}$

Distinguishing athlete's heart from HCM may require consideration of the broader HCM phenotype when LV wall thickness is in the range of 13-15 $\mathrm{mm}$ (i.e. the 'gray-zone'). ${ }^{29-31}$ Useful criteria include (Table 2): enlarged LV cavity size ( $\geq 55 \mathrm{~mm}$, common in athletes, very rare in HCM), maximum LV wall thickness $>15 \mathrm{~mm}$ and asymmetric distribution of LV wall thickening (consistent with HCM), markedly enlarged left atrium (possible in athletes, common in $\mathrm{HCM}$ ). Left ventricle filling and relaxation patterns (with $\mathrm{e}^{\prime}$ peakvelocity $>11.5 \mathrm{~cm} / \mathrm{s}$ on tissue Doppler analysis) are consistently normal in athletes whereas diastolic function is often impaired in HCM.

Cardiac magnetic resonance imaging (CMR) provides better imaging quality and more accurate wall thickness measurements than echo in the distal LV and lateral wall, and is required for diagnosis particularly when LVH is completely (or predominantly) limited to anterior free wall, posterior septum or apex. Moreover, contrast-enhanced CMR with late gadolinium enhancement (LGE) can detect areas of myocardial fibrosis. Late gadolinium enhancement, often in a patchy, multifocal mid-myocardial distribution is common in HCM, particularly in the regions of hypertrophy.

Abnormal ECG patterns are common in HCM patients (up to $90 \%$ of probands) and may be present in advance of the appearance of imaging $\mathrm{LVH}^{32,33} \mathrm{~A}$ variety of ECG alterations have been reported in patients with $\mathrm{HCM}$, but no single pattern can be 


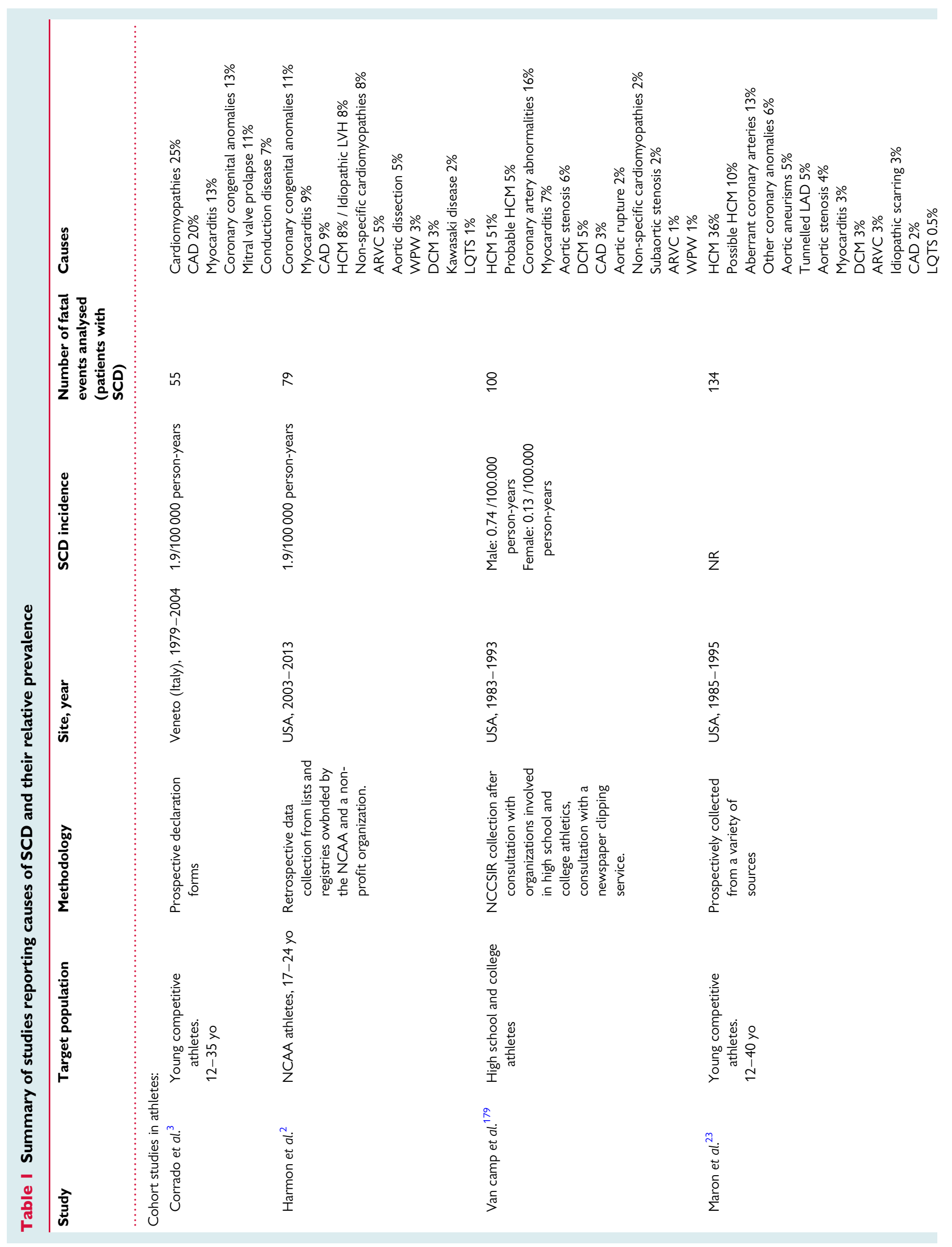




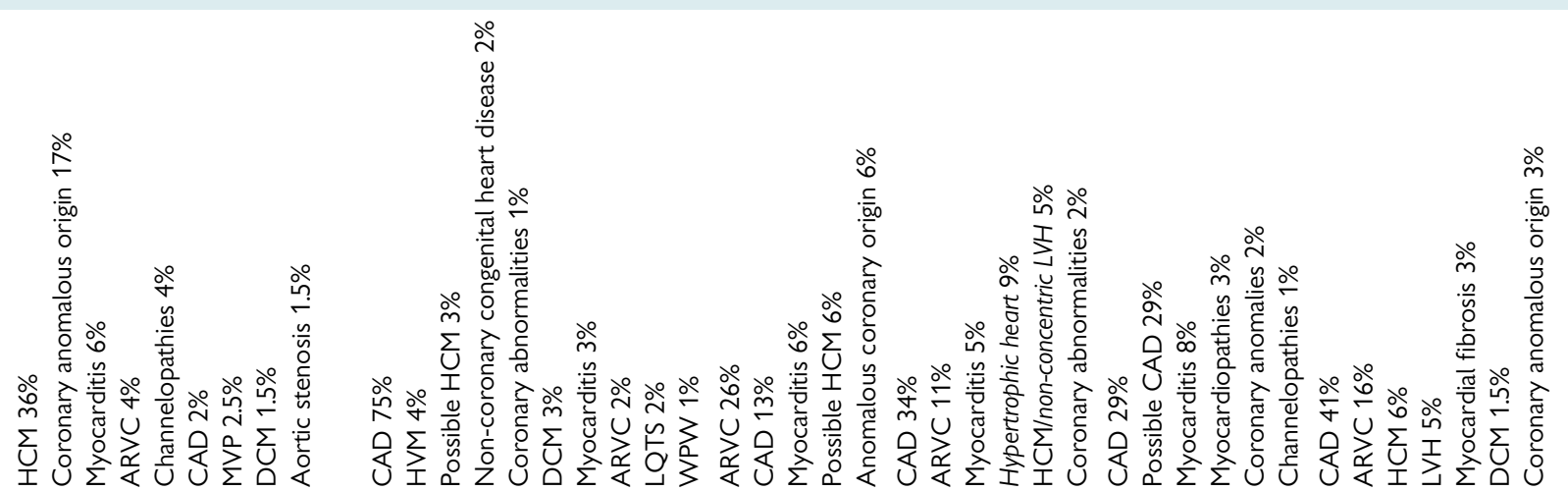

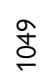

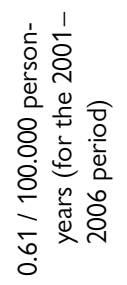

8
0
1
1
0
0
0
$\mathbb{1}$
3
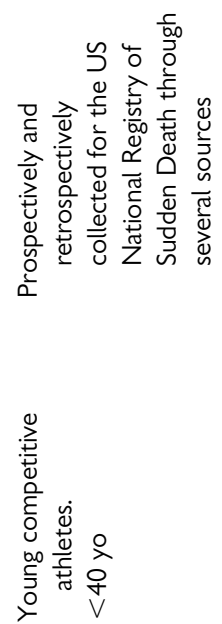

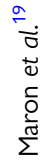

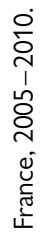

م
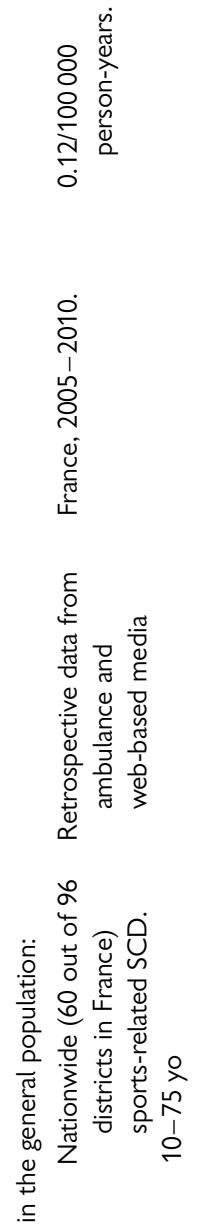

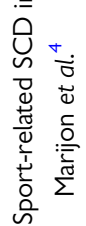

$\stackrel{n}{\sim}$

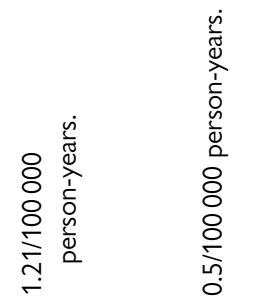

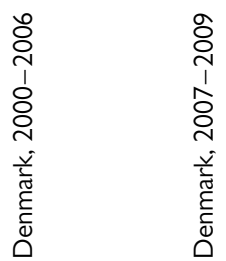

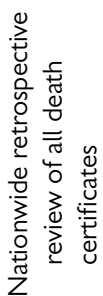

Ұ

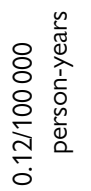

$\stackrel{\propto}{z}$

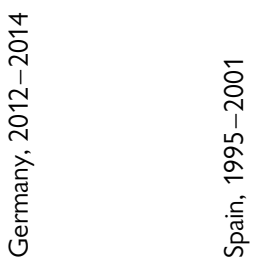

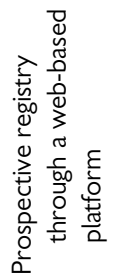
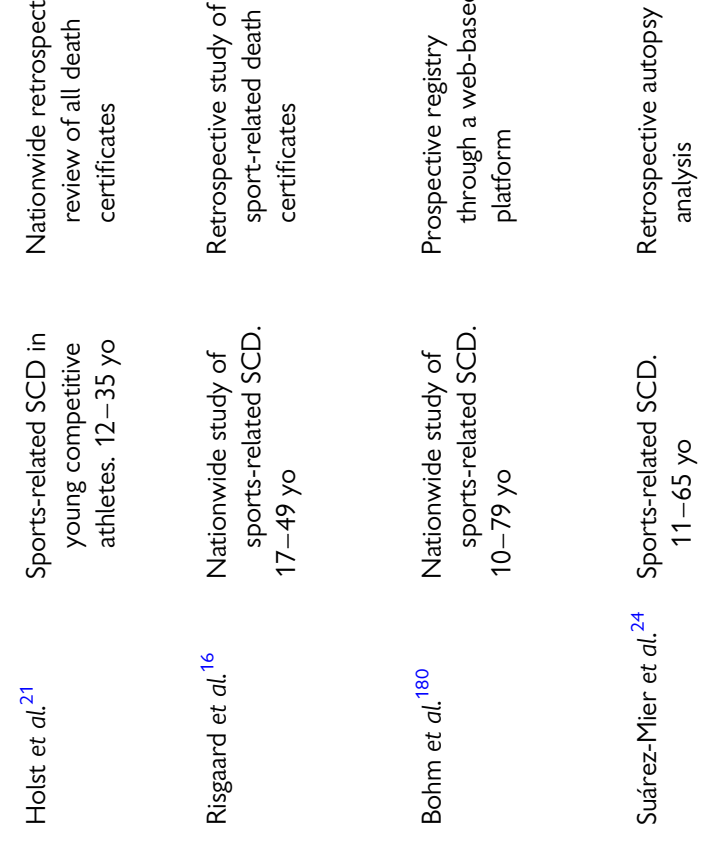

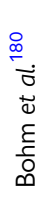

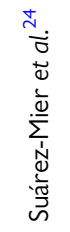


considered as the hallmark of the disease. However, certain abnormalities (e.g. T-wave inversion in inferior-lateral leads, pathological $\mathrm{Q}$ waves, left axis deviation,) usually represent the earliest and most sensitive disease manifestation in $\mathrm{HCM}^{32,33}$ Nevertheless, such changes may be seen in up to $5 \%$ of white and $15 \%$ of black elite athletes in the absence of diagnostic imaging abnormalities. ${ }^{34,35} \mathrm{Ap}$ propriate interpretation of such ECG abnormalities requires evaluation of the LV phenotypic features, the family background, type of athletic training, and in some instances the molecular genetics. ${ }^{35,36}$

Additional non-invasive testing to solve the differential diagnosis include assessment of exercise capacity and presence of occult arrhythmia (exercise test, $24 \mathrm{~h} \mathrm{ECG),} \mathrm{while} \mathrm{serial} \mathrm{imaging} \mathrm{tests} \mathrm{may}$ demonstrate the $\mathrm{LVH}$ regression in athletes after a period of complete deconditioning.

Genetic testing has been advocated for providing a definitive diagnosis of HCM in athletes with borderline LV wall thickening. However, interpretation of an identified genetic variant is often problematic and the yield for a positive genetic test result in the absence of a familial HCM does not exceed $20 \%$. Current guidelines recommend mutation analysis in certain borderline patients after a thorough assessment by specialized teams or when screening relatives has been planned. ${ }^{9}$

\section{Arrhythmogenic right ventricular cardiomyopathy}

Arrhythmogenic right ventricular cardiomyopathy (ARVC) is a progressive inherited heart muscle disease characterized by ventricular electrical instability which may lead to arrhythmic SCA, mostly in young people and athletes. ${ }^{37,38}$ Prevalence estimations range from 1 in 1000 to 1 in 5000 in the general population. ${ }^{10}$ Molecular genetic studies showed that ARVC is a genetic disorder resulting from defective desmosomal proteins, generally with an autosomal dominant inheritance. ${ }^{39,40}$ The pathological hallmark of the disease is the progressive loss of myocardium with subsequent myocyte death and fibro-fatty scar, which predisposes to life-threatening ventricular arrhythmias. $^{10,37,38,41}$

Exercise and sport in ARVC patients. Physical activity may trigger ventricular arrhythmias in some patients with ARVC. A retrospective

\section{Table 2 Factors supporting an athlete's heart diagnosis (vs HCM) in athletes with a LV hypertrophy in the 'grey zone' (i.e. LV wall thickness 13-15 mm)}

\footnotetext{
Supporting 'athlete's heart'

Clinical

Lack of HCM family history ${ }^{30}$

Absence of diffuse $\mathrm{T}$-wave inversion in $\mathrm{ECG}^{30}$

Cardiac magnetic resonance imaging

Homogenous distribution of LV hypertrophy

Lack of LGE areas in the LV (myocardial fibrosis)

Echocardiography

Normal or Enlarged LVEDD ( $\geq 55 \mathrm{~mm})$

Symmetric (IVS and PW, base to apex) LV hypertrophy

Normal diastolic function (E/A ratio $>1$; e' peak velocity $>11.5 \mathrm{~cm} / \mathrm{s}$ )

LV hypertrophy regression after complete deconditioning

Lack of known HCM-causing mutations in genetic testing
}

study suggested that competitive sports activity was associated with a five-fold increased SCD risk in adolescent and young adults with ARVC. ${ }^{5}$ Sport has been implicated as a factor promoting disease progression (Figure 1), ${ }^{42-44}$ but definitive confirmation is lacking. Genetically determined impairment of cell-to-cell adhesion may lead to myocyte death due to mechanical stress occurring during exercise and sports activity. ${ }^{42}$

Recent data suggest that an ARVC-like disease might be associated with intense endurance exercise in the absence of demonstrable desmosomal alterations; this form is addressed later in the 'Exercise as a promoter of cardiac injury' section.

Diagnosis: Diagnosis of ARVC is based on the criteria originally proposed by an International Task Force in the $1994,{ }^{45}$ later revised in $2010 .^{46}$ The diagnosis is based on combination of multiple sources of information, i.e. genetic, electrocardiographic, arrhythmic, morpho-functional, and histopathologic findings. ${ }^{46}$

The ECG is of particular value in raising suspicion for ARVC, in consideration that ECG abnormalities are present in the majority of patients (up to $80 \%$ ) (Figure 2). ${ }^{37,38,46-48}$ The most common abnormalities include prolonged QRS duration $\geq 110 \mathrm{~ms}$, inverted $\mathrm{T}$-waves in the right precordial leads $\left(\mathrm{V}_{1}-\mathrm{V}_{3}\right)$ or beyond in the absence of complete right bundle branch block in individuals $>14$ years old, or late potentials by signal averaged ECG in up to $74 \%$ of ARVC patients. ${ }^{46}$ Epsilon waves (low amplitude potentials at the end of QRS complex) are also common, but show a low interobserver reproducibility. ${ }^{49}$ Ventricular arrhythmias such as premature ventricular contractions (PVCs) or non-sustained/sustained ventricular tachycardia (NSVT) are relatively common in ARVC patients and typically present with a left bundle branch block pattern and either superior or inferior axis. ${ }^{37,38}$

Echocardiography, right ventricular (RV) angiography, and CMR may show a globally enlarged RV cavity, but wall motion abnormalities such as bulging and aneurysms in the RV wall may precede the RV dilation. ${ }^{50} \mathrm{CMR}$ also allows the identification of areas of altered signal intensity, consistent with fatty replacement, and ventricular LGE corresponding to fibrotic scars into the RV and/or the LV. Conversely, athletes (mostly engaged in endurance disciplines) usually present an enlarged RV in association with enlarged LV, with preserved global RV function and, most important, in the absence of segmental wall motion abnormalities. ${ }^{46}$ Not uncommonly, however, imaging testing may present only mild, if any, abnormality despite occurrence of clinically relevant tachyarrhythmias in ARVC patients. ${ }^{51}$

Genetic diagnosis of ARVC might be useful in certain cases for family screening purposes, as affected relatives might benefit from timely treatment and lifestyle modifications. ${ }^{39,52}$

\section{Coronary congenital abnormalities}

Coronary arteries arising from an anomalous sinus in the aortic root are the most common coronary artery congenital anomalies. Roughly $0.5-1 \%$ of individuals are affected, most frequently with a right coronary artery originating from the left coronary sinus. ${ }^{53}$ Most patients remain clinically silent and undiagnosed, while some will develop ventricular ischaemia and, a minority, present with $\mathrm{SCD}$ as a first manifestation. The risk of $\mathrm{CV}$ complications, including $S C D$, is higher in those patients with a left coronary artery originating in the right coronary sinus or those coronaries coursing between the aorta and pulmonary artery. ${ }^{53}$ 


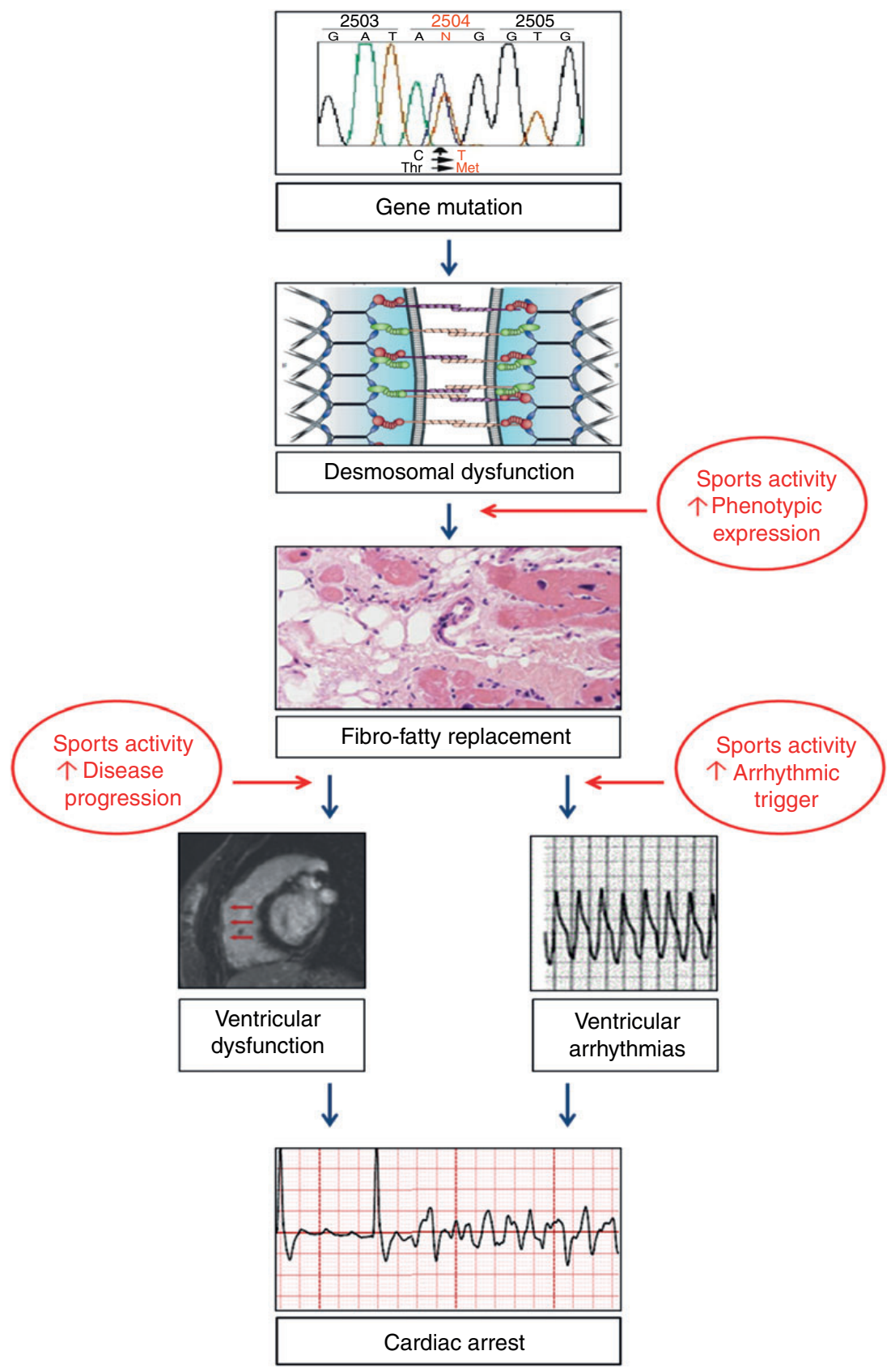

Figure I Schematic representation of ARVC course from desmosomal-gene mutation to phenotypic expression and cardiac arrest due to ventricular fibrillation. Sports activity may promote development of phenotypic expression, accelerate disease progression, and trigger lifethreatening ventricular arrhythmias.

Exercise and sport in individuals with coronary anomalous origins: Strenuous efforts might trigger ventricular ischaemia and electrical instability leading to VF. Some series point anomalous origin of a coronary artery as the second most common cause of SCD in athletes (Table 1). ${ }^{2,23}$ Coronary congenital abnormalities have been associated with a nearly 80 -fold increased risk of SD. ${ }^{3}$ Notably, nearly half of athletes who die because of coronary arteries with an anomalous origin were previously asymptomatic. ${ }^{54}$

Diagnosis: The diagnosis of a potentially lethal origin of a coronary requires a high degree of clinical suspicion. In the absence of a previous myocardial infarction, rest ECG is usually normal or show non-specific findings. Exercise test yields a low sensitivity for coronary abnormalities. ${ }^{54}$ In hands of expert sonographers, the origin of both (normal) coronaries can be localized in most individuals. ${ }^{55}$ A definite diagnosis is achieved with an invasive coronary or a computed tomography angiography.

\section{Ventricular pre-excitation}

Atrioventricular bypass tracts cause an early, anomalous ventricular activation that precedes normal activation through the AV node. 


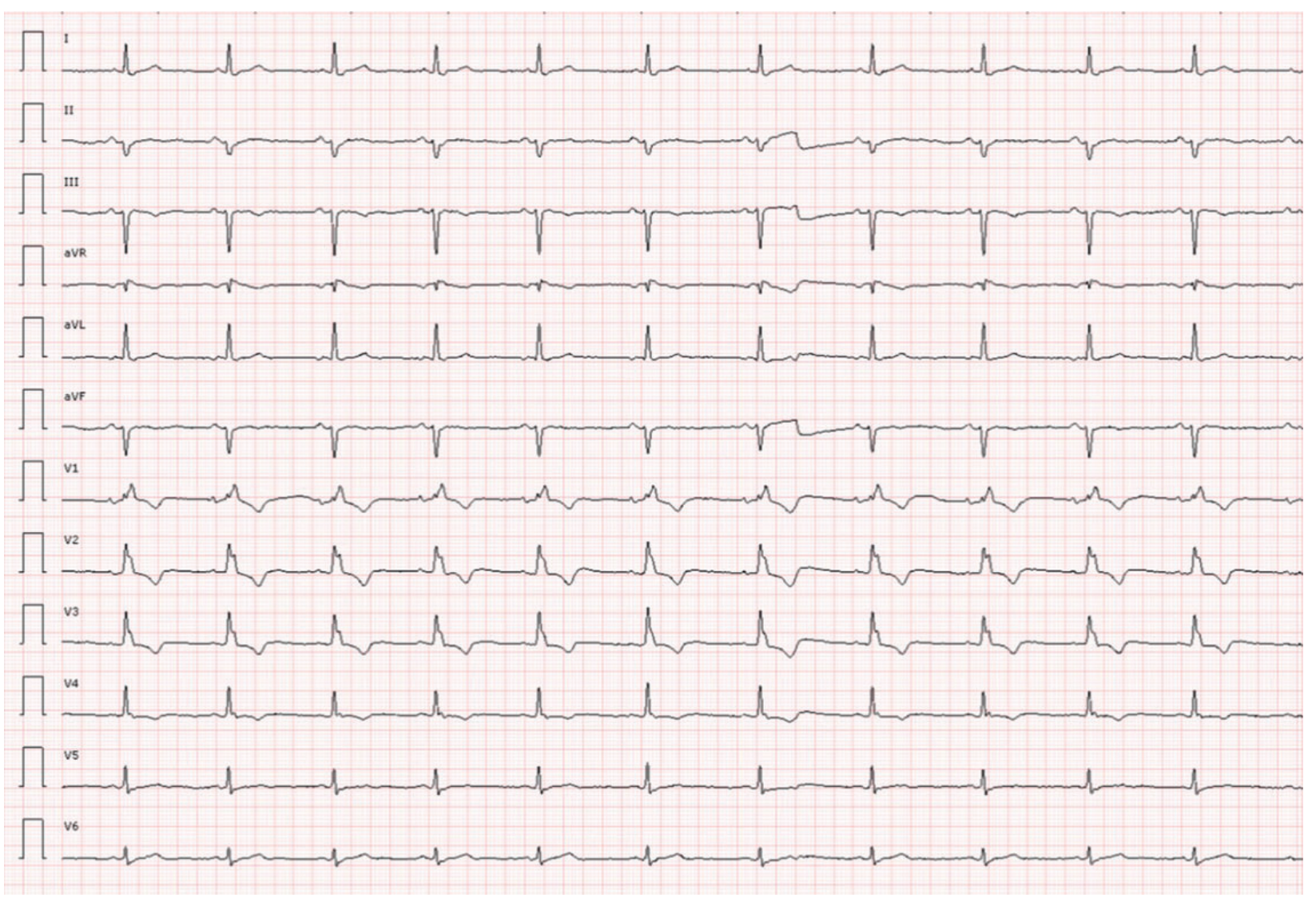

Figure 2 Representative ECG from an ARVC patient.

Accessory pathways generally lack decrementing properties (i.e. slower conduction at higher heart rates) and thus can be stimulated at very high rates. In certain circumstances with rapid atrial activation, such as in atrial fibrillation, an extremely high ventricular activation rate through the accessory pathway might trigger VT/VF and SCD. It has been estimated that 1:1000 individuals show ECG preexcitation patterns in the general population. ${ }^{56}$

Patients can remain asymptomatic or present with paroxysmal supraventricular tachycardia symptoms. Palpitations due to an orthodromic atrio-ventricular re-entry is the most common symptom in patients with ventricular pre-excitation. Syncope or presyncope might occur as a consequence of very rapid paroxysmal supraventricular tachycardias. While symptomatic patients are at a high risk of SCD $(\approx 0.15 \%$ per year), risk is lower in asymptomatic patients. A short refractory period of the accessory pathway has been proposed to identify those asymptomatic individuals with ventricular pre-excitation who are at risk of SCD.

Exercise and sport in individuals with ventricular pre-excitation: Very rapid ventricular activation during strenuous physical activity or $A F$ events has been suggested to trigger SCD in athletes. Because previous ECGs are often unavailable in athletes with SCD, a precise risk estimation is challenging. Ventricular pre-excitation is present in $\approx 1 \%$ of athletes with SCD (Table 1$).{ }^{19}$

Diagnosis: An ECG showing a characteristic delta-wave provides the diagnosis of ventricular pre-excitation (Figure 3). Slurred upstroke
QRS complexes along with short PR intervals ( $<120 \mathrm{mseg}$ ) characterize delta waves. Notably, ventricular pre-excitation may be occasionally masked by situations with a fast atrioventricular node conduction, such as in adrenergic settings.

\section{Left ventricle non-compaction}

Left ventricle non-compaction (LVNC) is a recently identified cardiomyopathy characterized by abnormal, prominent LV wall trabeculations and deep intertrabecular recesses, often associated with a thin compacted epicardial myocardial layer. ${ }^{10}$ It is a relatively uncommon condition, accounting for $<0.5 \%$ of all echocardiographic examinations. ${ }^{57}$ Up to half of patients show an X-linked or autosomal dominant inheritance pattern, with most mutations identified in genes encoding sarcomere proteins. ${ }^{58}$ Rather than an homogenous disease, several subtypes have been identified, including a dilated, an hypertrophic, a biventricular and a restrictive form. A benign form of LV hypertrabeculation, involving a normal cardiac function, no ventricular arrhythmias and no cardiac events at followup, has also been reported. ${ }^{59}$

Patients affected with LVNC might remain asymptomatic (particularly in benign forms) or present with symptoms derived from progressive LV systolic dysfunction, potentially lethal ventricular arrhythmias or thromboembolic events.

Exercise and sport in LVNC patients: The role of exercise in triggering arrhythmias or progression in LVNC patients is uncertain. Most 


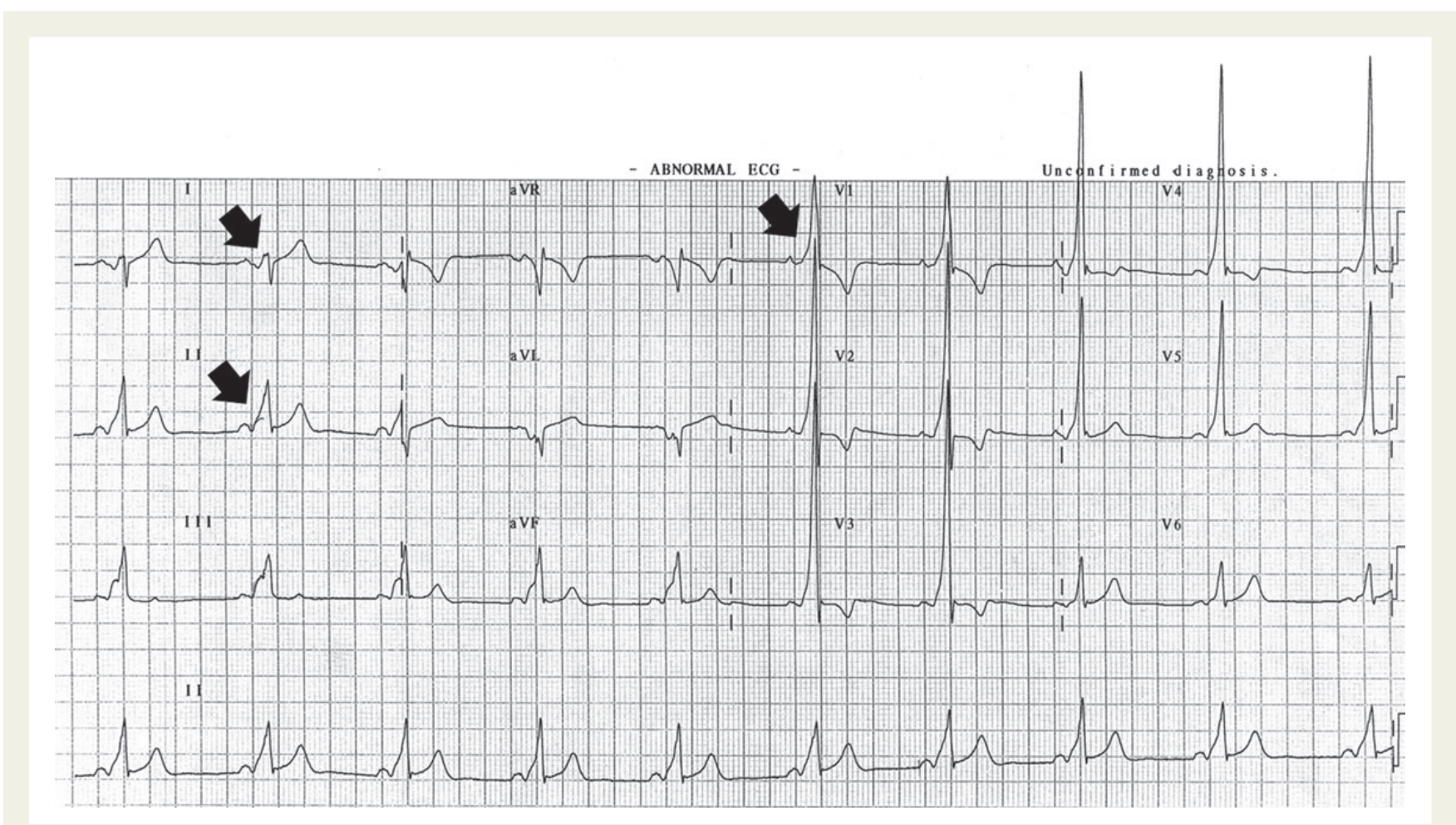

Figure 3 Representative ECG from a patient with a pre-excitation syndrome (arrows).

SCD series have not reported LVNC to be a significant cause of SCD in athletes.

Diagnosis: Unspecific findings are common in the ECG. ${ }^{58,60}$ A definite LVNC diagnosis is obtained by mean of imaging techniques showing prominent trabecula. The most widely used echocardiographic criterion relies on the non-compaction to compaction ratio (usually 2:1) to ascertain a LVNC diagnosis, although alternative criteria have been proposed. ${ }^{58}$

Recent reports suggest an increased LV trabecular pattern in athletes engaged in a variety of sports disciplines, eventually fulfilling LVNC criteria in up to $8 \%$ of athletes. ${ }^{61}$ Factors such as normal systolic function, absence of ECG abnormalities, absence of complex ventricular arrhythmias, and a negative family history support a benign clinical outcome in these athletes. ${ }^{60}$

\section{Dilated cardiomyopathy}

Dilated cardiomyopathy (DCM) is currently defined by the presence of LV or biventricular dilatation and systolic dysfunction in the absence of abnormal loading conditions (hypertension, valve disease) or coronary artery disease sufficient to cause global systolic impairment. ${ }^{62}$ Due to a relatively preserved cardiac output in many cases, patients may remain asymptomatic over a long course of their disease. Symptoms usually relate to reduced cardiac output; however, lifethreatening ventricular and/or supraventricular tachyarrhythmias are not uncommonly part of the initial clinical presentation.

Exercise and sport in DCM patients: An increase in the adrenergic tone and hemodynamic overload during physical activity can trigger ventricular arrhythmias. Nevertheless, DCM is a relatively uncommon cause of SCD in athletes (Table 1). 2,16,19,23,63
Diagnosis: An imaging technique commonly provides a definitive diagnosis by showing a disproportionately dilated LV cavity, usually with a spherical shape, systolic dysfunction (i.e. ejection fraction $<50 \%$, with blunted increase during exercise), and occasionally accompanied by wall motion abnormalities. In trained athletes, LV cavity enlargement may be similar, but maintains an ellipsoid shape with a normally positioned mitral valve, and is associated with an enlargement of right ventricular and atrial chambers. Indeed, LV function remains normal (i.e. ejection fraction $>50 \%$, with normal increase during exercise) and wall motion abnormalities are absent in athletes. $^{64}$

Non-specific changes in the ECG, such as repolarization abnormalities, left bundle branch block, and ventricular or supraventricular tachyarrhythmias are often found in patients with DCM.

\section{Valvulopathies}

Valve regurgitation and stenosis are commonly degenerative and most frequently found in aged individuals. Nevertheless, bicuspid aortic valve and mitral valve prolapse are particularly important in young and middle-aged individuals. Bicuspid aortic valves are found in $\approx 1 \%$ of the general population ${ }^{65}$ and pose the affected individual at risk for aortic stenosis or regurgitation, ascending aorta dilation and dissection over time. Mitral valve prolapse is the most common cause of primary mitral regurgitation in young to middle-aged individuals. When severe, valvulopathies superimpose a hemodynamic overload that might lead to LV dysfunction, ventricular arrhythmias and an inadequate increase in cardiac output during physical activity.

Exercise and sport in patients with valvular heart diseases: There is no data showing that exercise accelerates valvulopathy progression. 
Nevertheless, physical activity may trigger SCD in some athletes with valvular heart diseases, particularly if hemodynamically significant. Valvulopathies are not uncommonly found amongst the most frequent causes of SCD in athletes (Table 1).

Diagnosis: A careful physical examination usually reveals a cardiac murmur in patients with a significant valvulopathy. A definite diagnosis is provided by a cardiac imaging test; an echocardiography usually informs of the type of valvulopathy, its severity and mechanisms.

\section{Inherited primary arrhythmia syndromes}

Inherited primary arrhythmia syndromes are genetically determined, primary arrhythmic disorders in the absence of a clinically apparent, overt structural heart disease. The most frequent entities are long and short QT syndrome, catecholaminergic polymorphic ventricular tachycardia, and Brugada syndrome. ${ }^{10,66}$

\section{Long QT syndrome}

Long QT Syndrome (LQTS) is a collective name used to identify a group of disorders characterized by an increased duration of ventricular repolarization, represented by a prolonged QT interval on surface electrocardiogram, and by the predisposition to develop life-threatening ventricular arrhythmias, characteristically Torsade de pointes ventricular arrhythmias during exercise or stressful circumstances. An estimated LQTS prevalence of 1 out of 2000 newborns has been reported. ${ }^{67}$

Mutations in 15 genes encoding for cardiac ion channels have been associated with LQTS. ${ }^{68}$ Genetic screening identifies a disease-causing mutation in about $75 \%$ of LQTS cases. ${ }^{69}$ In most families, LQTS inheritance follows an autosomal dominant pattern [either isolated QT-prolongation, also known as Romano-Ward syndrome (LQT1-6; LQT9-13), or associated with extra-cardiac manifestations (LQT7-8)]. Homozygous or compound heterozygous mutated forms of KCNE1 or KCNQ1 associate with profound congenital deafness in the autosomal-recessive inherited Jervell and Lange-Nielsen syndrome. ${ }^{69-73}$

Exercise and sport in LQTS patients: Physical activity and emotional stressors act as triggers for ventricular arrhythmias and SCD in patients with LQTS. The role of sport activity, primarily swimming, is particularly relevant for LQT1 patients, but a significant overlap exists amongst all genotypes. ${ }^{69,73}$

Diagnosis: The diagnosis of LQTS relies on the measurement of the QT interval corrected for heart rate (QTc) using Bazett's formula on repeated 12-lead electrocardiograms at stable heart rates between 60 and 100 bpm (Figure 4). According to the latest diagnostic criteria, ${ }^{10}$ LQTS should be considered in patients with an unexplained syncope and a QTc $>460 \mathrm{~ms}$. In asymptomatic individuals without a pathogenic mutation or a family history of LQTS, a QTc $\geq 480 \mathrm{~ms}$ is required to establish the positive diagnosis.

When QT prolongation is less obvious, a score combining the age of the patient, clinical and family history, the QTc duration, T-wave morphology, and previous history of Torsade de pointes has been created $^{74}$ : a LQTS risk score $>3$ is also considered diagnostic for LQTS. An accurate clinical history, physical exam, blood test or other tests as needed should aim at ruling out secondary causes of QT prolongation (e.g. bundle branch block, drugs, electrolyte imbalance, cardiac ischaemia).
Additional clues for the diagnosis of LQTS derive from the dynamic analysis of QT interval under different circumstances and stimuli. An exercise test allows exploring the adaptation of the QT interval to rapidly increasing heart rates, which has been demonstrated to be impaired in LQTS, ${ }^{75}$ especially LQT1. Interestingly, LQT1 and LQT2 patients also show an exaggerated and paradoxical QT prolongation after physical effort. ${ }^{76,77}$ A QTc inter$\mathrm{val} \geq 480 \mathrm{~ms}$ at the fourth minute of recovery from exercise stress test has been recently suggested as a new criterion to consider in the LQTS risk score ${ }^{78}$ after demonstrating $100 \%$ specificity in identifying LQT1 and LQT2 patients. ${ }^{79}$ Similarly, QT also fails to adapt to sinus tachycardia after standing up quickly in LQTS patients and may elicit an exaggerated QTc prolongation; ${ }^{80}$ a QTc $>499$ at maximal nearness between the end of the $T$-wave and the beginning of the following P-wave (i.e. maximal stretching) shows a $90 \%$ sensitivity and $87 \%$ specificity for the diagnosis of LQTS. ${ }^{81} \mathrm{~A}$ 12-lead ambulatory ECG is recommended to comprehensively evaluate the QTC interval in precordial leads at different times throughout the day, ${ }^{10}$ including the night when LQT3 patients exhibit the largest QTc prolongation. ${ }^{82}$ Eventually, a LQTS diagnosis should always be confirmed by a specialized cardiologist.

Up to $1 / 3$ of carriers of a pathogenic mutation exhibit normal QTc values on resting ECG (incomplete penetrance) ${ }^{83}$ and, according to the guidelines, ${ }^{10}$ should be considered as 'affected' by LQTS.

Aside from the prolonged QT interval, LQTS patients often present with additional ECG features including excessive bradycardia for the age, visible T-wave alternans or notches on the T-wave (suggestive for LQT2); long sinus pauses are common among LQT3 patients.

\section{Short QT syndrome}

Short QT syndrome (SQTS) is a term that identifies patients with reduced repolarization duration that pose affected individuals at risk of life-threating arrhythmias and SCD. Mutations in five genes encoding ion channel subunits have been linked to SQTS pathology, but $80 \%$ of cases remain gene-elusive. Although initial reports pointed to an extremely high arrhythmic risk, unselected cohorts of patients with a short QT suggest a significantly lower risk. $^{84,85}$

The prevalence of short QT, as defined with actual guidelines, has been estimated at 1:2000 in paediatric individuals, but at about $1: 1000$ in adult populations including athletes, ${ }^{85,86}$ with a consistent predominance for males.

Exercise and sport in SQTS patients: No specific triggers for STQS arrhythmic events have been identified, and thus the consequences of strenuous physical activity in SQTS patients remain unknown.

Diagnosis: The diagnosis of SQTS is established in those patients with a shorter than normal QTc (Figure 5). Nevertheless, an accurate threshold to identify those patients with a short repolarization that pose at risk of SCD remains controversial. Current ESC guidelines propose a QTc $\leq 340 \mathrm{~ms}^{10}$ to diagnose SQTS. Diagnosis should be considered in the presence of a QTc $\leq 360 \mathrm{~ms}$ in the secondary prevention setting as well as for patients with a familiar history of SCD or SQTS or in patients with a known causing mutation. Notably, secondary causes of short QT (e.g. increased plasma potassium or calcium concentration, acidosis and hyperthermia) should be ruled out. 


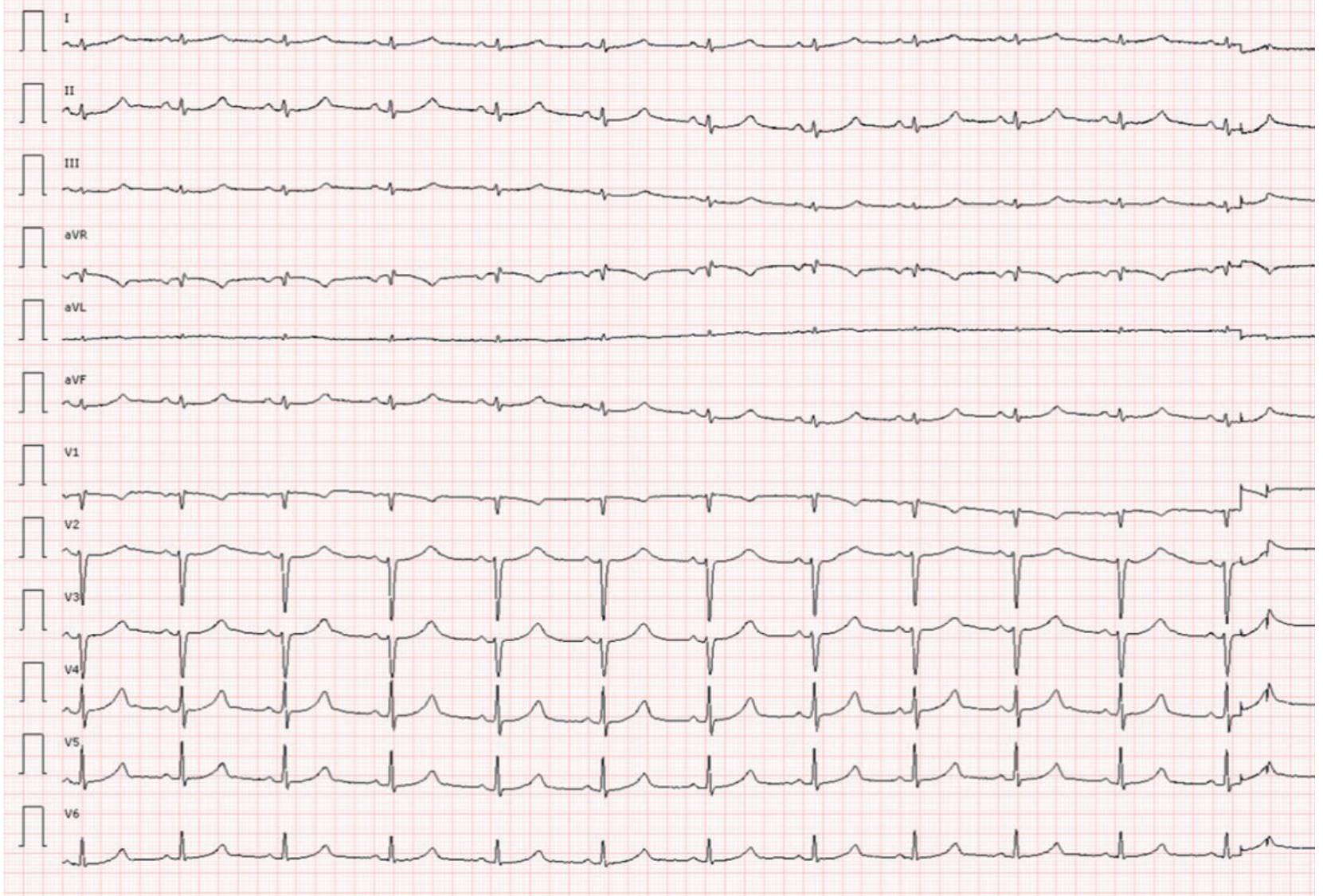

Figure 4 Representative ECG from a LQTS patient. Note a markedly prolonged cQT interval (510 msec).

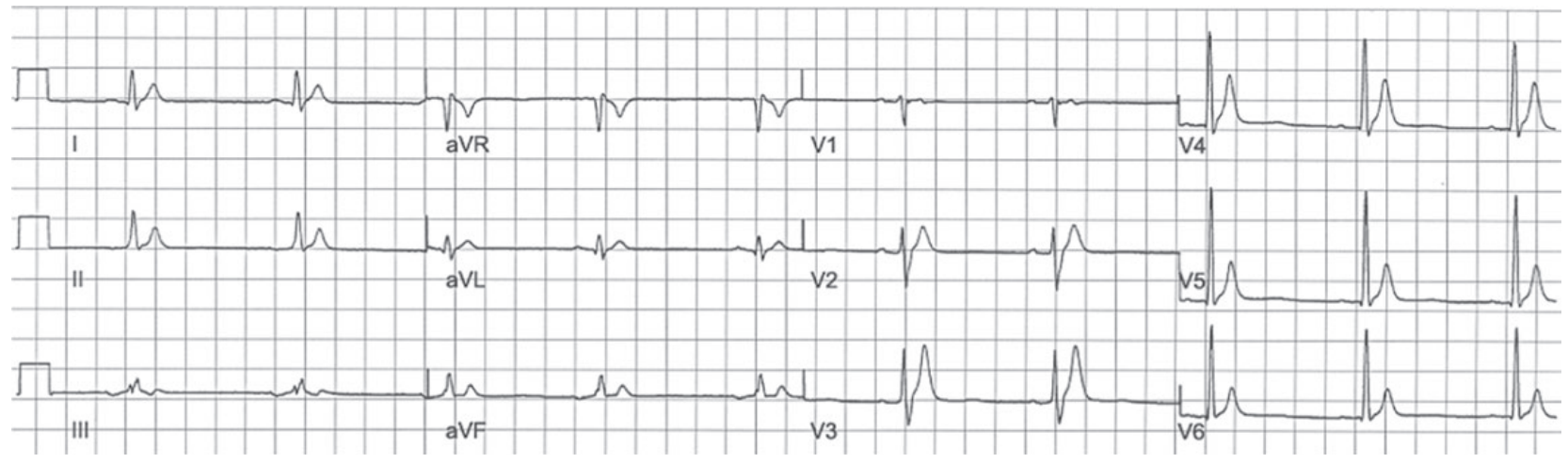

Figure 5 Representative ECG from a SQTS patient. Note a markedly shortened cQT interval (260 msec).

\section{Catecholaminergic polymorphic ventricular tachycardia}

Catecholaminergic polymorphic ventricular tachycardia (CPVT) is a severe disease characterized by adrenergic-induced bidirectional or polymorphic ventricular tachycardia. The prevalence of the disease is unknown, with the most commonly quoted estimate being 1:10 000 .
Two main genetic variants of CPVT are known, accounting for about two thirds of cases: ${ }^{11}$ an autosomal dominant one, secondary to mutations in the RyR2 gene ( $~ 55 \%$ of cases) and an autosomal recessive one, stemming from genetic defects in the CASQ2 gene (about $5 \%$ of cases). Mutations in both proteins alter calcium 
homeostasis in cardiac myocytes, facilitating diastolic calcium release that leads to the development of delayed-after-depolarizations and triggered activity that induce supraventricular and ventricular arrhythmias.

When unrecognized, CPVT has an extremely poor prognosis, with untreated mortality rates of $30-50 \%$ by age $40 .^{11,87}$

Exercise and sport in CPVT patients: Physical activity is a common trigger for adrenergically mediated ventricular arrhythmias and SCD in CPVT patients.

Diagnosis: One of the major difficulties in recognizing CPVT is that patients show normal resting ECGs. Lower-than-normal heart rates and prominent $U$ waves have been reported in some patients, but they are not specific for a diagnosis.

According to the latest diagnostic criteria, ${ }^{10} \mathrm{CPVT}$ is diagnosed in the presence of a structurally normal heart, normal ECG, and effortor emotion-induced ventricular tachycardia. Typically, exercise stress testing or strong emotions elicit the appearance of VPBs that increase in complexity with the heart rate, ending with the pathognomonic bi-directional or polymorphic ventricular tachycardia. The term 'bi-directional' refers to the $180^{\circ}$ rotation on the frontal plane of the QRS complexes of the ectopic beats. Adrenergically mediated supraventricular arrhythmias (premature atrial contractions, runs of supraventricular tachycardia and bursts of atrial fibrillation) are also frequent in CPVT. The use of catecholamine infusions has been suggested as a possible alternative to exercise, but its sensitivity is not clearly defined. ${ }^{11,88}$

CPVT is a highly penetrant disease and the prevalence of 'silent' mutation carriers is estimated at $20 \%,{ }^{87}$ but cardiac events may still occur in this subset of individuals, who therefore need to be treated according to current guidelines. ${ }^{10,89}$ In these patients genetic testing is the pivotal diagnostic tool. ${ }^{87}$

\section{Brugada syndrome}

The term 'Brugada syndrome' (BrS) identifies a clinical setting characterized by 'right bundle branch block, persistent ST segment elevation and sudden death' due to polymorphic ventricular tachycardia (PVT) and/or ventricular fibrillation (VF) in the absence of other cardiomyopathies. ${ }^{90}$ Although it was initially identified as a purely electrical disease in the absence of structural heart disease, recent data suggest that a CMR exam may uncover minor RV abnormalities. ${ }^{91}$

The $\mathrm{BrS}$ is an inherited disease with an autosomal dominant pattern of transmission and variable penetrance. However, up to $60 \%$ of cases may be sporadic; that is, absent in other relatives. ${ }^{92,93}$ Prevalence in the general population ranges from 1 in 1000 in Asia to less than 1 in 10000 individuals in Europe and America. ${ }^{94}$

Exercise and sport in Brugada syndrome patients: There is a scarcity of large-scale or prospective data on the safety of sports participation in individuals with $\mathrm{BrS}$. Malignant ventricular arrhythmias generally occur at rest after an increased vagal activity and/or withdrawal of sympathetic activity. Thus, increased vagal predominance as a consequence of chronic athletic conditioning might eventually enhance the propensity of athletes with $\mathrm{BrS}$ to die at rest or during exercise recovery. Furthermore, elevation of body temperature during high-intensity efforts could potentially trigger fatal arrhythmias in these patients.
Diagnosis: The BrS is diagnosed when a type $1 \mathrm{ST}$-elevation is observed either spontaneously or after intravenous administration of a sodium channel blocker in at least one right precordial lead (V1 and $\mathrm{V} 2$ ), placed in a standard or superior position (up to the second intercostal space) (Figure 6). Other findings which support and might accompany the diagnosis of $\mathrm{BrS}$ include attenuation of ST elevation during maximal exercise with reappearance during the recovery phase (although in selected patients carrying a SCN5A mutation the ST elevation may become more evident with exercise); ${ }^{95,96}$ firstdegree atrio-ventricular block and left axis deviation; atrial fibrillation; late potentials in high resolution ECG; fragmentation of the QRS; ventricular refractory period $<200 \mathrm{~ms}$ and an $\mathrm{HV}$ interval $>60 \mathrm{~ms}$ in an electrophysiological study; and absence of a diagnostic for an alternative cardiomyopathy.

Other causes of ST-segment elevation such as the early repolarization pattern often seen in young athletes or the use of psychotropic and anti-allergic agents should be excluded before establishing the diagnosis of $\mathrm{BrS}$. On the other hand, certain modulating factors can unmask or exacerbate the typical $\mathrm{BrS}$ pattern. Fever modulates the phenotype by accentuating the sodium channel inactivation, unmasking the type 1 ECG pattern and triggering ventricular arrhythmias. Exposure to some other drugs or electrolyte imbalance may also produce ST elevation suggestive of BrS, likely related to a possible genetic predisposition.

\section{Acquired cardiac abnormalities}

In addition to aforementioned inherited cardiac conditions, acquired heart disease, including coronary artery disease, valvular disease or myocarditis, may also increase the risk of SCD. Acquired heart diseases becomes proportionally more important in older athletes.

\section{Coronary artery disease}

Exercise and sport in patients with (known/silent) coronary artery disease: Atherosclerotic coronary artery disease (CAD) is the predominant cause of unexpected SCD in the general population. Vigorous physical activity increases the risk of both SCD and acute myocardial infarction (AMI) relative to less vigorous activity. ${ }^{97}$ The relative risk of an exercise-associated SCD or AMI is greatest in the most sedentary individuals and decreases with increasing level of regular physical activity. The absolute risk of an exercise-associated AMI has not been determined, but approximately $10 \%$ of AMls may be related to physical activity. ${ }^{98}$ Notably, approximately $30 \%$ of sport-associated SCD victims experienced typical symptoms of cardiac ischaemia in the week prior to their event. ${ }^{63}$

Most acute cardiac events in previously asymptomatic individuals occur from acute plaque rupture or erosion with acute thrombosis in coronary arteries not previously hemodynamically compromised. ${ }^{97}$ At least one series of SCD during running events, however, noted that all of the 5 SCD victims with CAD had advanced atherosclerotic disease but none had evidence of acute thrombosis. ${ }^{1}$

Diagnosis: Rest ECG appears to be a non-accurate test for detecting CAD. Whether or not to routinely screen with a 12-lead ECG in all master athletes remains debated. American Heart Association guidelines on assessing CAD risk in athletes and active individuals recommend exercise testing prior to the initiation of vigorous exercise training in those at high risk of coronary events, including men 


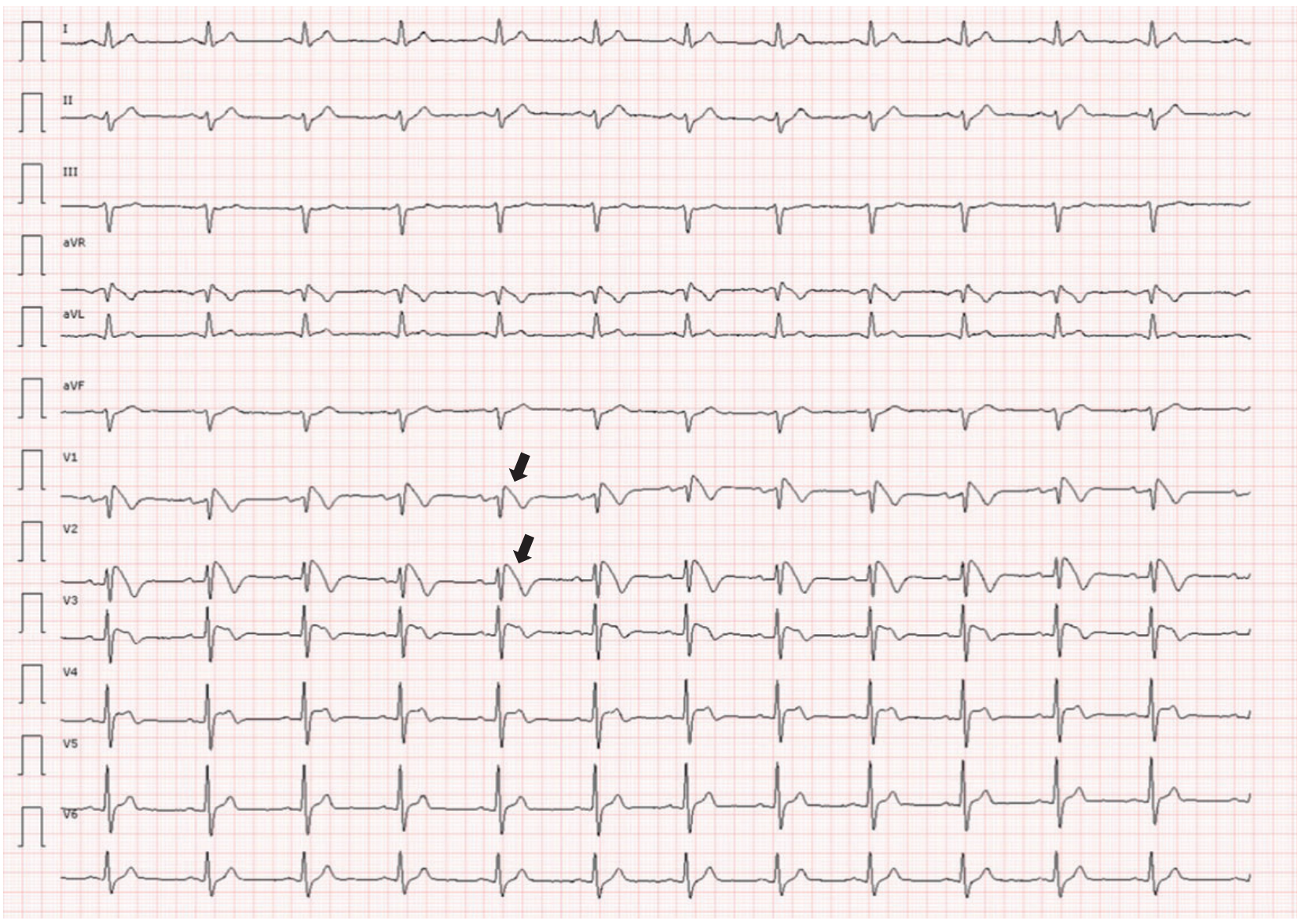

Figure 6 Representative ECG showing characteristic findings from Brugada syndrome (arrows).

$>40$ years of age and women $>50$ years of age (or postmenopausal) with one or more coronary risk factors. ${ }^{99}$ European professional society recommendations for PPE in master athletes approach this issue by assessing the cardiovascular risk profile (by a validated risk score form) of the candidate and the type and intensity of athletic discipline [European Association for Cardiovascular Prevention and Rehabilitation (EACPR)]. ${ }^{8}$ However, there are no randomized, controlled clinical trials evaluating these recommendations.

With actual evidence, senior athletes $\left(\geq 35\right.$ years old $\left.{ }^{8}\right)$ and individuals participating in vigorous physical or sport activity should:

(1) Be informed of the nature of cardiac prodromal symptoms and their need for prompt medical evaluation.

(2) Undergo exercise stress testing if symptoms of possible CAD are present.

(3) An exercise stress test might be considered in senior athletes at a high cardiovascular risk, although conflicting data exists on the efficacy of such approach.

\section{Chagas disease}

Chagas heart disease $(\mathrm{ChD})$ remains the most important cause of non-ischaemic cardiomyopathy in Latin America and may become a global health problem due to emigration. ${ }^{100} \mathrm{ChD}$ usually occurs in $20-30 \%$ of the infected individuals and clinical manifestations appear 10-30 years after the initial infection. ${ }^{101,102}$ Cardiac arrhythmias and SCD are frequent and may occur any time of the disease evolution, even in individuals without overt cardiomyopathy. ${ }^{100-104}$

Scar-related sustained VT is the main cause of SCD in patients with ChD. Global or segmental LV dysfunction predicts SD occurrence, particularly if associated with syncope or non-sustained VT during ambulatory Holter monitoring or stress testing. ${ }^{105-107}$ Moreover, sinus node dysfunction, AV and intra-ventricular conduction disturbances are common findings on ECGs of asymptomatic patients with ChD, but can progress to sinus node standstill and complete AV block, causing syncope and eventually creating the conditions to SCD. ${ }^{101-103}$

Exercise and sport in ChD patients: Limited evidence suggests that athletes with ChD are at risk of SCD during intense exercise. ${ }^{108}$ The absence of symptoms does not exclude the presence of cardiomyopathy even in high performance athletes.

Diagnosis: Chagas disease diagnosis requires an initial epidemiological inquiry (origin from endemic areas of Chagas disease) and subsequent serologic tests. Clinical warning signs (PVCs; AV and IV conduction disturbances) and symptoms (unexpected decrease in physical capacity, syncope or near syncope) may indicate cardiac disease. Echocardiography (where LV dysfunction indicates high 
SCD risk), $24 \mathrm{~h}$ ECG recording and exercise test (PVCs and NSVT suggest high risk), CMR (LV/RV LGE), and potentially an electrophysiological study (inducible sustained VT) might serve to stratify SCD risk.

\section{Myocarditis}

Myocarditis identifies myocardial involvement in the setting of systemic inflammatory processes, histologically evidenced by inflammatory infiltrates and myocyte degeneration and necrosis in the absence of an ischaemic origin. Myocarditis signs and symptoms shows a high interindividual variability, ranging from minor regional wall motion abnormalities or pericardial effusion to severe LV systolic dysfunction and potentially lethal ventricular arrhythmias. Although some cardiac manifestations are temporal and will regress over variable periods of time, some will remain as a myocarditis sequela (e.g. in the form of DCM).

Exercise and sports in patients with myocarditis: Myocarditis is a common finding in SCD series in athletes (Table 1) but, as later addressed, vigorous physical activity might also accelerate and worsen myocardial affectation in systemic viral and autoimmune processes.

Diagnosis: Myocarditis should be suspected in those individuals with systemic viral symptoms, including upper respiratory tract or gastrointestinal manifestations, who develop cardiac-related symptoms such as heart failure, arrhythmias, or chest pain. Myocardial necrosis markers are commonly increased in plasma in early stages. ECG commonly shows unspecific signs such as ST-segment and T-wave abnormalities, or ventricular arrhythmias. Cardiac imaging techniques might show global or regional wall motion abnormalities along with variable degrees of pericardial effusion. Myocardial oedema might be identified in early stages with CMR, while LGE patches can be present early in its evolution and remain as a sequela. Myocardial biopsy provides a definitive diagnosis, but it is usually not required.

\section{Exercise as a promoter of cardiac injury}

In addition to acting as a trigger for arrhythmic events, there are three ways in which intense and prolonged exertion may impact on the risk of SCD by promoting the disease substrate itself:

(a) Exercise may accelerate an inherited predisposition to cardiomyopathy: There are data in both murine models ${ }^{109,110}$ and humans with desmosomal mutations demonstrating more rapid phenotypic ARVC expression with RV dysfunction, arrhythmias and progression to cardiac transplant or death in those who perform regular strenuous exercise. ${ }^{42,43}$ Similar exercise/genotype interactions are largely unexplored in other inherited conditions. There is some preclinical data in HCM to suggest that exercise may favourably modify cardiac remodelling but the relevance of these findings to human disease is not yet known. ${ }^{111}$

(b) Exercise by itself might cause arrhythmogenic remodelling: Data in recent years suggest that intensive and chronic exercise training promotes arrhythmogenic remodelling, especially of the RV, in the absence of a demonstrable genetic predisposition. The 'exercise-induced RV cardiomyopathy' as defined by Heidbuchel et al. is based on the clinical observation that professional cyclists presenting with RV arrhythmias very seldom show clinical or genetic evidence of inherited disease. ${ }^{112,113}$ An endurance-trained rat model confirms a selective, arrhythmogenic deleterious effect of high-intensity training in the RV. ${ }^{114}$ Endurance athletes are over-represented in 'gene-elusive' ARVC cohorts ${ }^{115}$ which raises the possibility that a still undefined (poly)genetic predisposition other than a typical desmosomal mutation may predispose to deleterious RV remodelling associated with intense and prolonged exercise training. There is substantive mechanistic data supporting disproportionate strain on the RV during intense exercise. ${ }^{16,117}$ Moreover, some endurance athletes demonstrate markedly enlarged hearts with subnormal function. ${ }^{118}$

(c) Exercise may exacerbate the severity of cardiac damage due to intercurrent illness: A recent concept is that intense exercise may exacerbate cardiac damage due to a concurrent illness such as myocarditis, i.e. that the hemodynamic overload of intense exercise may impair healing or even exacerbate myocardial inflammation. There is theoretical rationale for this concept and some limited evidence from animal studies. ${ }^{119}$ Also the clinical observation in athletes of an unanticipated prevalence of large subepicardial fibrotic regions, most likely due to post-myocarditis scarring, is interpreted along these lines. ${ }^{120}$

The possibility of the evolving nature of structural disease in athletes which can be promoted or worsened by regular exercise implies that serial cardiac pre-participation evaluation need to be considered, especially in at-risk groups, such as those engaged in most intense exercise (cyclists, rowers, marathon runners etc.) and/or after an overt viral illness.

\section{Role of screening techniques}

\section{Role of electrocardiogram}

The inclusion of an ECG in addition to a medical history and physical examination has focused most debates on the most appropriate PPE protocol to identify athletes at risk for SCD. Pre-participation CV evaluation that relies solely on medical history (personal and family) and physical examination has a limited ability to identify potentially lethal CV abnormalities in young athletes, because cardiac conditions responsible for SCD are often clinically silent and unlikely to be suspected or diagnosed on the basis of spontaneous symptoms. ${ }^{48}$ The addition of 12-lead ECG substantially enhances the power of screening for early detection of athletes with cardiomyopathies or channelopathies, which commonly manifest with ECG abnormalities ${ }^{48,121}$ : sensitivity improves from less than $25 \%$ for clinical history and exam to $>90 \%$ for ECG. ${ }^{122}$ A recent meta-analysis of available studies comparing history, physical examination, and ECG demonstrated that the most effective strategy to detect underlying (predisposing to SCD/SCA) disease is ECG. ${ }^{122}$

Figure 7 summarizes the arguments in favour and against including the 12 lead ECG at rest for the pre-participation CV evaluation.

Practical considerations and limitations of the ECG: There are a number of considerations and limitations to ECG screening, from the clinical and technical to psychological, ethical, and economic view that merit further discussion. 


\section{Arguments in favour of ECG}

- A 12 lead basal ECG is a non-expensive, largely available test for a mass screening of athletes.

\section{- The ECG screening modality has proven more sensitive than the history and physical examination protocol. ${ }^{117}$}

- ECG is abnormal in $>80 \%$ of individuals with cardiomyopathies (hypertrophic cardiomyopathy ${ }^{31,32}$ and arrhythmogenic right ventricular cardiomyopathy ${ }^{36}$ ), myocarditis, pre-excitation syndromes, and ion channel disorders which are the leading causes of cardiac arrest in young athletes. Together these conditions account for up to the two-thirds of SCD in young competitive athletes.

- Modern diagnostic criteria offer the potential to standardise and simplify the interpretation of ECGs and improve diagnostic accuracy. ${ }^{121}$

- There has been a notable decline ( $90 \%$ ) of SCD among young competitive athletes after implementation of the nationwide Italian ECG screening programme over 25 years of observation. ${ }^{3}$

\section{Arguments against ECG}

- The evidence for a survival benefit of ECG screening is limited to a single observational study.

- Highly trained athletes may develop ECG anomalies mimicking cardiomyopathies causing SCD. ${ }^{33,34}$

- Misinterpretation of 12 lead ECG is not uncommon particularly in non-specialized physicians, ${ }^{126}$ leading to an expensive diagnostic work-up or unnecessarily disqualifying athletes because of normal ECG variants; conversely, potentially lethal heart disease may be misinterpreted as normal variants of the athlete's ECG.

- Some cardiovascular disorders at risk of sudden death are not associated with ECG changes (Marfan syndrome, premature coronary heart disease, congenital coronary anomaly ${ }^{53}$ ).

- False-positive results yield a high number of work-up tests that accounts its high cost and jeopardizes cost effectiveness of ECG screening programmes. ${ }^{163-168}$

- Abnormal ECG findings lead to disqualification of up to $2 \%$ of athletes from competitive sports. ${ }^{3}$

Figure 7 Pros and cons of ECG screening.

The most important clinical limitation of ECG screening lies in imperfect sensitivity and specificity. Among the most common causes of SCD in athletes, sensitivity of the ECG for HCM is $90 \%{ }^{126}$ but coronary artery anomalies, the second most common cause of SCD in athletes in some series, are most often silent on ECG. ${ }^{54}$ Similarly, the sensitivity of the rest ECG to identify ischaemic heart disease, the most common cause of SCD in athletes older than 35 years, remains rather low. Even in HCM, the ECG may not show abnormalities until adolescence or later. ECG manifestations of channelopathies such as LQTS and Brugada vary over time, while CPVT is electrically silent at rest. However, it should be noted that the history and physical exploration also lack sensitivity, as symptoms may be under-reported, and reported symptoms such as dyspnoea or reduced physical performance may be unspecific and difficult to interpret. $^{127}$

Similarly, specificity is imperfect, with ECG screening generating false positive rates of $5-10 \%,{ }^{128}$ leading to either unnecessary secondary evaluations or restriction from sports activity. It is notable that the false positive rate for ECG screening is largely affected by the criteria used to define 'abnormalities'. Significant advances have been made in our understanding of how to interpret an athlete's ECG. Modern standards have been proposed to distinguish physiologic cardiac adaptations in athletes from findings suggestive of underlying pathology, namely the European Society of Cardiology criteria $^{129}$ or more recent 'Seattle criteria'130 (Figure 8). Studies applying these modern ECG criteria have resulted in substantially lower false positive rates, ${ }^{123,131,132}$ with new proposals refining the interpretation of athlete's ECG. ${ }^{36,133}$

Technical limitations include the need for widespread expertise in both the acquisition and interpretation of the ECG. Numerous studies show that even cardiologists have imperfect expertise in interpretation of ECGs. ${ }^{134,135}$ Training-related changes can vary by age, sex, race, ${ }^{136}$ ethnicity, and type of sport, ${ }^{34}$ further complicating appropriate interpretation. Use of standardized criteria ${ }^{129,130}$ can improve interpretation, and educational modules have been created and are largely implemented (i.e. http://learning.bmj.com/learning/ course-intro/.html?courseld=10042239, and the 'JS Sports ECG' app, available for Android and iPhone smartphones). The history and physical examination however are also imperfectly applied, often without appropriate training. ${ }^{137}$ Efforts to improve training in the PPE history and physical (http://www.njleg.state.nj.us/2014/ Bills/S0500/471_I1.HTM) are also key to adequate screening. In this regard, a curriculum core for sports cardiology qualification has been proposed. ${ }^{138}$

Concerns regarding the psychological impact of athlete ECG screening have also been raised. ${ }^{139}$ However, two studies which have actually measured psychological outcomes in athletes undergoing ECG screening programmes ${ }^{140,141}$ have found that athletes reported feeling more confident, would recommend screening to others and distress was very low. Moreover, those athletes whose screening included ECG were more satisfied and positive about the screening than those who received only history and physical examination, including those with initially false positive results.

Ethical concerns have also been raised about the fairness of screening just a subset of the overall population, in consideration that also young people not participating in athletics have a risk of SCD. ${ }^{128,142}$ Some reports show that athletes are even protected from SCD in comparison to the general population, ${ }^{21}$ a fact not reproduced by others. ${ }^{5}$ In one study of US high school students, the annual incidence of sudden cardiac arrest was 1.14 per 100000 


\section{ECG findings in athletes}

\section{Physiological adaptation \\ Do not require further revaluation}

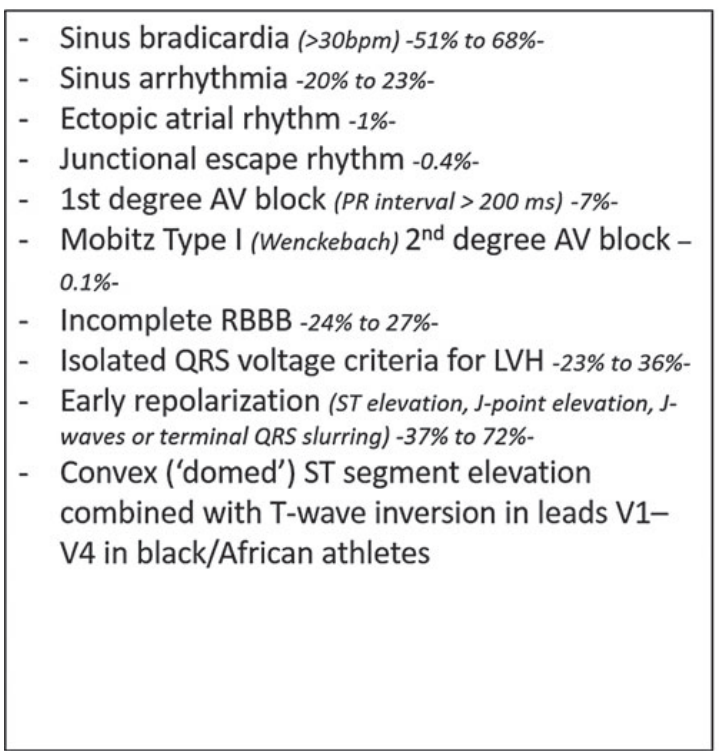

Abnormal ECG findings

Require further evaluation

\begin{tabular}{|c|}
\hline 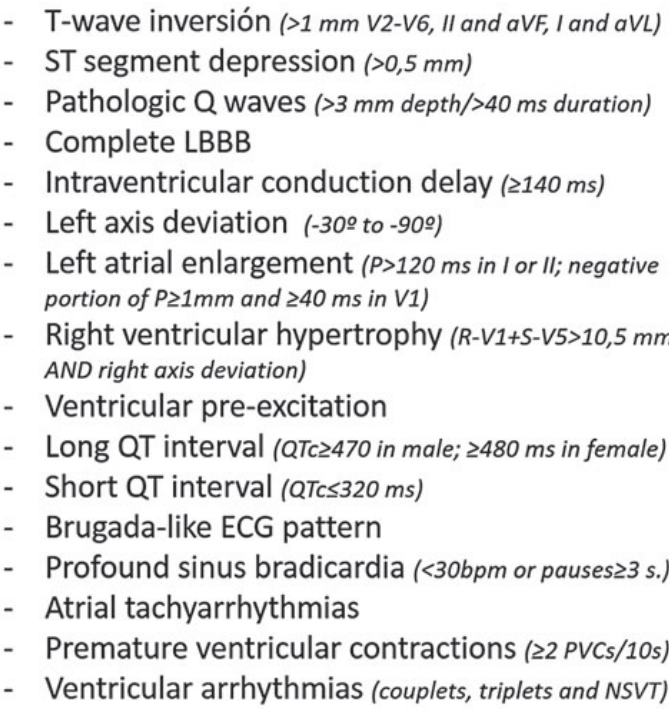 \\
\hline
\end{tabular}

Figure 8 Classification of ECG abnormalities in the athlete according to European Society of Cardiology recommendations. Common ECG abnormalities (group 1): up to $80 \%$ of trained athletes exhibit ECG changes that are the consequence of the physiologic cardiovascular adaptation to sustained physical exertion and do not reflect the presence of an underlying cardiovascular disease; an estimate of frequency in heterogeneous cohorts of athletes is shown. ${ }^{123-125}$ Uncommon ECG abnormalities (group 2) are unrelated to athletic conditioning and should be regarded as an expression of possible underlying cardiovascular disorders, notably cardiomyopathies and cardiac ion channel diseases. LBBB, left bundle branch block; RBBB: Right bundle branch block; LVH: Left ventricular hypertrophy.

for athletes, vs. 0.31 for student non-athletes. ${ }^{15}$ Similarly, a previous investigation in Italian population showed that young athletes are exposed to a 2.8-fold increased risk for SCD in comparison to nonathletes. ${ }^{1}$ Whether this potential difference in absolute risk warrants different approaches to risk-reduction in the athlete and non-athlete can be debated. In some countries, all children undergo ECG screening in school, ${ }^{143}$ and in others, incorporation of the ECG into routine paediatric care has also been shown to be feasible. ${ }^{144}$

It is important to note that each of these concerns-sensitivity, specificity, lack of expertise, the ethical impact, and cost-all apply similarly, and in many cases more so, to the use of the history and physical exploration assessment. ${ }^{6,128}$

\section{Role of ECG exercise test}

Exercise test is most often used to unveil myocardial ischaemia and detect CAD. The diagnostic yield of exercise testing largely relies on the pre-test ischaemia probability. Most positive exercise tests in asymptomatic individuals are falsely positive and require additional testing to exclude disease. A decision analysis evaluation of exercise testing before regular exercise concluded that 'the number of exercise-induced deaths prevented (by exercise testing) is less than the added number of deaths from angiography. ${ }^{145}$ Overall, mortality in this report was only reduced for those individuals at moderate to high CAD risk undergoing exercise test. ${ }^{145}$ Moreover, limited sensitivity further jeopardizes the role of ECG exercise test as a mass screening tool. A classic meta-analysis reported a $68 \%$ sensitivity for exercise ECG test, meaning that one in three patients with CAD will show normal; specificity remains below $80 \% .{ }^{146}$ In summary, ECG exercise test leads to misleading conclusions and increases costs when used as a mass screening tool; conversely it should be reserved for symptomatic athletes or those deemed to be at high risk of CAD.

However, it is here emphasized that exercise testing indications in athletes during a PPE are not limited to the CAD diagnosis, but may also be useful to evaluate the blood pressure behaviour and the occurrence of arrhythmias, to evaluate symptoms occurring during physical activity, and to assess the physical performance and its progression in relation to exercise training and competitive sport participation. As previously stated, exercise test might also serve in the diagnostic or therapeutic approach of LQTS or CPVT patients in some instances.

\section{Role of ambulatory ECG monitoring}

Prolonged recordings provide data on ECG over extended and variable periods of time, allowing assessment of ECG during normal day life. Available tools include classic techniques such as short, 24 h or 7 day ECG Holter recordings or implantable devices lasting for $>2$ years, to modern tools such as smartphone or wearable apps that allow easily obtaining short ECG recordings in almost any situation. 
The diagnostic efficiency of ambulatory ECG recordings in firstline PPE is limited because of a very low sensitivity, and therefore remains a second-line test. The most common indications for ambulatory ECG monitoring are unexplained syncope and palpitations. It may also be considered to explore bradyarrhythmias or to quantify PVC density after initial PPE tests. Specific applications for ambulatory ECG recordings such as QT assessment in patients with suspected LQTS should also be considered.

The diagnostic yield is highly dependent on the frequency of symptoms and duration of the ECG recording. Infrequent symptoms are rarely reproduced during a 24 or $48 \mathrm{~h}$ recording and show a very low sensitivity; longer recording-periods with implantable recorders might better fit this need. In some other instances (e.g. PVC quantification) $24 \mathrm{~h}$ recordings might suffice.

\section{Role of imaging modalities in screening}

Imaging modalities have been established as an integral part of the evaluation of athletes who are flagged by the initial PPE as potential carriers of cardiac disease. ${ }^{35,147}$ Transthoracic echocardiography (TTE) and CMR will identify the majority of athletes with cardiomyopathy; coronary angiography utilizing computed tomography (CTCA) or CMR are the non-invasive modalities of choice for coronary artery anomalies; and coronary artery calcium scoring (CACS), CTCA, and stress imaging are employed for the investigation of athletes with suspected atheromatous coronary artery disease.

While some studies have evaluated TTE as a first-line massscreening tool, CMR, CTCA, and CACS are almost exclusively used to assess athletes with symptoms or abnormalities found on first-line screening tests.

\section{Echocardiography}

The inherent limitations of the 12-lead ECG as a screening tool for structural heart disease has prompted a number of sporting organizations to advocate TTE as part of the screening protocol for the most elite of their athletes. Transthoracic echocardiography is the only imaging modality that has been evaluated in the context of mass PPE as it is widely available, portable, free of ionizing radiation and of relatively low cost. Investigators have employed a number of screening protocols in an attempt to optimize the diagnostic utility of mass TTE for conditions predisposing to SCD in athletes, while minimizing time and costs. Protocols range from 1 min targeted visualization of the parasternal views to a $20 \mathrm{~min}$ comprehensive echocardiographic study.

Feinstein et al. ${ }^{148}$ and Weidenbener et al. ${ }^{149}$ employed limited TTE utilizing the long and short axis parasternal views. The studies focused on two-dimensional assessment of the left cardiac chambers in an attempt to identify athletes with hypertrophic cardiomyopathy, Marfan's syndrome, aortic stenosis, or mitral valve prolapse. Most TTE studies were completed within 2 min. Both studies failed to identify any athlete with quiescent cardiomyopathy. The majority (78\%) of abnormalities identified by TTE (in a relatively small cohort of athletes) related to congenital valvular abnormalities and in particular bicuspid aortic valve and mitral valve prolapse. ${ }^{149}$ Wyman et $a .^{55}$ employed a more comprehensive 5 min screening protocol, which included colour Doppler and velocities. Similar to the earlier studies, minor and trivial valvular abnormalities were the predominant finding. Of importance, the authors were able to identify the origin of both coronary arteries in $96 \%$ of the athletes.

Contemporary studies in healthy children and young athletes have reiterated that the addition of TTE as a first-line screening tool to the 12-lead ECG does not increase the diagnostic yield of cardiomyopathies. ${ }^{36,144,150,151}$ In addition, elite athletes may exhibit cavity dilatation and LV hypertrophy that overlaps with mild or incomplete phenotypes of cardiomyopathies, raising concerns that mass TTE may result in further increase of the false positive screening rate. ${ }^{35,61,64,152,153}$

An alternative PPE protocol, which advocates performing TTE as an intermediate step to individuals who are flagged by history, physical examination or ECG for further evaluation has been proposed. The use of onsite TTE reduces referral rates by $40-60 \%$ and has a significant positive impact on associated costs, use of resources and time delays until the athlete is cleared for competition. ${ }^{150}$

In summary, the value of TTE as a first-line screening tool for conditions predisposing to SCD remains to be proven, largely because of additional cost, lack of expertise and infrastructure, and no evidence for incremental diagnostic value compared to ECG alone.

\section{Cardiac magnetic resonance imaging}

As compared with echocardiography, CMR enables better assessment of the morphology and function of all cardiac chambers whilst also enabling assessment of acute oedema and myocardial fibrosis. ${ }^{154}$ Recent developments suggest that CMR may also become a mean for assessing cardiac function in athletes during exercise. ${ }^{155}$ It has the distinct advantage of being able to provide high-quality images in virtually all subjects and providing detail of regions of the heart, which are difficult to image with echocardiography. In particular, CMR enables greater visualization of the LV apex and RV, regions of particular interest in athletes in whom apical HCM or ARVC are suspected.

CMR can identify pathology in athletes when the ECG and echocardiography appear to be normal, particularly when LGE techniques are used for tissue characterization. ${ }^{126}$ Thus, CMR should be considered the most comprehensive imaging modality for the exclusion of cardiac structural pathology in athletes with concerning symptoms or PPE abnormalities. However, the relative expense and limited availability of CMR make it less suitable for broad-based screening. Furthermore, the specificity of CMR testing in athletes has not been systematically evaluated and there is a possibility of a high burden of false positive results given the increased sensitivity for detecting variation in patterns of ' LV hypertrabeculation' or $\mathrm{RV}$ dilation. As an example, small patches of LGE have been reported in a significant minority of ostensibly healthy athletes, ${ }^{156,157}$ whilst in other athletes LGE seems to be associated with serious arrhythmias. ${ }^{120}$ With uncertainty regarding the significance of LGE and subsequent management in asymptomatic athletes, CMR is not recommended as a first-line screening modality.

\section{Computed tomography [...] coronary angiography and coronary artery calcium scoring}

CTCA is an excellent technique for assessing athletes in whom ischaemic heart disease is suspected, either due to anomalous coronary ostia or acquired atherosclerotic disease. The ability to identify the course of the coronary arteries relative to surrounding vessels 
and structures makes it the technique of choice for diagnosing anomalies in the coronary circulation and evaluating the prognostic implications. However, because significant coronary anomalies are relatively rare (less than 1 in $2000^{158}$ ), CTCA should be reserved for those athletes with symptoms or abnormalities on PPE, which raise justified clinical suspicion.

A second issue is that of acquired coronary artery disease, which constitutes the dominant cause of SCD in athletes aged in their 4th decade and beyond. Whilst intense exercise is an established trigger of coronary events, regular exercise training attenuates this risk. ${ }^{159}$ The rationale for screening for atherosclerotic coronary disease in master athletes is that the identification of at-risk subjects may lead to more aggressive preventative therapies, although the role of exercise promotion or restriction in these settings is unclear. Coronary artery calcium score is an established marker of cardiovascular risk ${ }^{160}$ but it remains uncertain as to whether resultant changes in management may significantly modify cardiovascular risk and future incidence of coronary events. Current guidelines support the use of CACS in males and females who have an intermediate cardiovascular risk (10-20\% Framingham risk score), in whom the result may be expected to change the outcome. Although there is some speculation that intense exercise training may impact on this risk, ${ }^{161,162}$ the evidence is not sufficiently compelling to suggest that screening recommendations should differ for athletes as compared with the wider population. Thus, CACS can be considered in the minority of middle-aged and older athletes with a coronary risk that is at least moderate in athletes in whom a justified clinical suspicion exists.

\section{Existing screening programmes, rationale, and limitations}

Cardiovascular PPE has increasingly been integrated into the annual pre-competition medical assessment of competitive athletes. Preparticipation evaluation attempts to identify pre-existing cardiac abnormalities, ensuring optimal management, and thereby reducing the potential for adverse events and loss of life, i.e. SCA/SCD. Nevertheless, the aim of PPE might not be limited to the identification of underlying cardiac conditions, but may represent a unique opportunity to establish contact with a physician, undertake a general health assessment and provide positive basic lifestyle habits.

Both the American Heart Association (AHA)/American College of Cardiology (ACC) ${ }^{163}$ and the European Cardiology Society (ESC) ${ }^{7}$ agree in that compelling justification exists for CV pre-participation screening on medical, ethical and legal ground. Whilst agreement on the implementation of PPE exists, there remains a difference in opinion regarding the methods employed to assess cardiac risk.

For competitive or professional athletes, a consensus statement of two Working Groups of the ESC recommends personal history, physical examination and a resting 12-lead ECG at a minimum, ${ }^{7}$ whilst the $\mathrm{AHA} / \mathrm{ACC}^{163}$ do not recommend the inclusion of the ECG in mass screening. This major difference ultimately impacts upon the sensitivity, specificity, and cost-effectiveness of the screening programme.

To protect athlete health, major international sporting bodies have taken the lead, either adopting the $\mathrm{ESC}^{7}$ or $\mathrm{AHA} / \mathrm{ACC}^{163}$ protocols or establishing their own, such as Fédération Internationale de Football Association (FIFA). Furthermore, the question of who gets screened is governed by the international sporting bodies themselves, rather than state sponsored healthcare systems (excluding Italy and Israel). For example, FIFA and Union of European Football Associations (UEFA), now mandate pre-tournament cardiac screening irrespective of which country an athlete originates from, while others, such as the International Olympic Committee (IOC), simply recommend it as the best practice. ${ }^{164,165}$

Table 3 shows the recommendation policy and methods of the screening protocols currently implemented by nineteen major international sporting organizations.

\section{Evidence supporting preparticipation evaluation programmes: impact on cardiovascular mortality}

The only evidence supporting that CV PPE is lifesaving is based on the Italian experience. ${ }^{3}$ Mortality data derived from a time-trend analysis over a 26 year period (1979-2004), when PPE was introduced, demonstrated that the incidence of SCDs in athletes decreased by 89\%; from 3.6 per 100000 person-years in the pre-screening period to 0.4 per 100000 person-years in the screening period. By comparison, the incidence of SCDs in an unscreened non-athletic population of the same age did not change over the same time duration. Death by the cardiomyopathies were greatly reduced, with the proportion of athletes identified and disqualified due to HCM and ARVC doubling from the early to the late screening period.

This study suggests that systematic PPE programme can significantly decrease mortality, via identification and disqualification of athletes with an underlying and unsuspected cardiomyopathy. This conclusion is supported by the following considerations; (i) the decrease in athlete deaths occurred at the same time as the implementation of national screening programme; (ii) the reduced mortality was largely due to fewer deaths from cardiomyopathies, which was accompanied by an increased number of cardiomyopathies identified at screening; (iii) the incidence of SCDs did not change among the unscreened non-athletic population of the same age.

Nevertheless, some limitations of the Italian work should be acknowledged, ${ }^{166,167}$ including its observational, retrospective design subject to an immortality-time bias. ${ }^{168}$ The fact that only fatalities were considered, thus ignoring a likely increasing number of successfully resuscitated cardiac arrests over the last decades might have created the impression that the incidence of cardiac arrest is decreasing when only fatal events are counted. ${ }^{169}$ Finally, some authors claim that a relatively high mortality rate observed at the beginning of mandatory ECG screening in Italy might have increased the odds of detecting a mortality reduction during the study period. $^{167,170}$

To date, the results of the Italian experience have not been reproduced by other research groups. The major criticism is based on the Israel experience. ${ }^{167}$ Steinvil et al. claim that the yearly incidence of athletic field deaths did not change between 1985-96 (2.54 per 100000 persons) and 1997-2009 (2.66 per 100000 persons) in Israel despite the introduction of a screening programme including 12-Lead ECG. However, major methodological limitations are also present in the Steinvil et al. ${ }^{167}$ work. First, the number of SCDs were derived from searching uniquely in two newspapers, largely underreporting cardiac events. ${ }^{2,14}$ Second, the size of the 
Table 3 Major international sporting federations with a pre-participation cardiovascular screening policy for elite or professional athletes

\begin{tabular}{|c|c|c|c|c|c|c|}
\hline \multirow[t]{2}{*}{ Sporting Federation } & \multirow{2}{*}{$\begin{array}{l}\text { Mandatory or } \\
\text { recommended }\end{array}$} & \multirow{2}{*}{$\begin{array}{l}\text { Screening } \\
\text { protocol }\end{array}$} & \multicolumn{4}{|c|}{ Primary screening methodology } \\
\hline & & & $\begin{array}{l}\text { Physical } \\
\text { examination }\end{array}$ & $\begin{array}{l}\text { Personal } \\
\text { symptom and FH }\end{array}$ & $\begin{array}{l}\text { 12-lead } \\
\text { ECG }\end{array}$ & Echocardiography \\
\hline International Olympic Committee & Recommend & $1 O C$ & $\checkmark$ & $\checkmark$ & $\checkmark$ & \\
\hline International Paralympic Committee & Recommend & IOC & $\checkmark$ & $\checkmark$ & $\checkmark$ & \\
\hline $\begin{array}{l}\text { Fédération Internationale de Football } \\
\text { Association }\end{array}$ & Mandate & Self-imposed & $\checkmark$ & $\checkmark$ & $\checkmark$ & $\checkmark$ \\
\hline Union of European Football Associations & Mandate & Self-imposed & $\checkmark$ & $\checkmark$ & $\checkmark$ & $\checkmark$ \\
\hline Union Cycliste Internationale & Mandate & Self-imposed & $\checkmark$ & $\checkmark$ & $\checkmark$ & $\checkmark$ \\
\hline $\begin{array}{l}\text { Fédératoin Internationale de } \\
\text { Motocyclisme }\end{array}$ & Mandate & Self-imposed & $\checkmark$ & $\checkmark$ & $\checkmark$ & $\checkmark$ \\
\hline $\begin{array}{l}\text { Fédération Internationale de } \\
\text { l'Automobile }\end{array}$ & Mandate & Self-imposed & $\checkmark$ & $\checkmark$ & $\checkmark$ & $\checkmark$ \\
\hline $\begin{array}{l}\text { International Association of Athletic } \\
\text { Federations }\end{array}$ & Recommend & $10 C$ & $\checkmark$ & $\checkmark$ & $\checkmark$ & \\
\hline National Basketball Association (USA) & Recommend & $\mathrm{AHA}$ & $\checkmark$ & $\checkmark$ & $\checkmark$ & \\
\hline National Football League (USA) & Recommend & $\mathrm{AHA}$ & $\checkmark$ & $\checkmark$ & & \\
\hline Major League Baseball (USA) & Recommend & $\mathrm{AHA}$ & $\checkmark$ & $\checkmark$ & & \\
\hline National Hockey League (USA) & Recommend & $\mathrm{AHA}$ & $\checkmark$ & $\checkmark$ & & \\
\hline World Rugby & Recommend & Self-imposed & $\checkmark$ & $\checkmark$ & $\checkmark$ & \\
\hline Fédération Internationale de Natation & Recommend & Self-imposed & $\checkmark$ & $\checkmark$ & $\checkmark$ & \\
\hline World Boxing Federation & Recommend & Self-imposed & $\checkmark$ & $\checkmark$ & $\checkmark$ & \\
\hline International Handball Federation & Recommend & IOC & $\checkmark$ & $\checkmark$ & $\checkmark$ & \\
\hline International Triathlon Union & Recommend & IOC & $\checkmark$ & $\checkmark$ & $\checkmark$ & \\
\hline Fédération Internationale de Ski & Recommend & $10 C$ & $\checkmark$ & $\checkmark$ & $\checkmark$ & \\
\hline $\begin{array}{l}\text { Fédération International des Sociétés } \\
\text { d'Aviron }\end{array}$ & Mandate & IOC & $\checkmark$ & $\checkmark$ & $\checkmark$ & \\
\hline
\end{tabular}

population of competitive athletes was not known, but rather estimated.

Maron et al. ${ }^{170}$ compared deaths rates in young athletes from Veneto screened with ECG vs. athletes from Minnesota (a demographically similar population) screened by history and physical examination, i.e. without ECG. They found that over a comparable 11 year period (1993-2004), 12 deaths were reported in Veneto and 11 in Minnesota. When analysed as deaths per 100000 personyears, the two regions did not differ significantly for the period $1993-2004$ ( 0.87 vs. 0.93 , respectively, $P=0.88$ ). The study concluded that SCDs in young competitive athletes occurred at a low rate in both Veneto and Minnesota, despite different screening strategies. However, also in this study caution is needed, in consideration that the numerator (i.e. SCDs) in American athletes was derived uniquely from insurance claims and media reports, methods known to underreport young athlete's events. ${ }^{2,14}$

\section{Cost-effectiveness considerations}

There is general agreement that ECG screening improves the sensitivity for detecting unsuspected cardiac disease among asymptomatic athletes. However, whether its additive cost within a preventive programme is financially sustainable remains largely controversial and dependent on the funding organ.
A calculation of the ECG-based cost-effectiveness was provided by Halkin et al., ${ }^{171}$ based on the Italian experience ${ }^{3}$ and assuming its generalizability to other regions. The following assumptions were made:

(1) The number of athletes undergoing medical screening remains constant because a $2 \%$ yearly increment in the population of athletes compensates for the $2 \%$ per year disqualification rates for athletes (as reported in the Italian study). ${ }^{3}$

(2) The entire population of athletes undergo repeated screening that includes history taking, physical examination, and ECG every single year. In addition, $9 \%$ of athletes require additional tests because of positive findings in the basic screening.

(3) For every 10000 athletes, it was estimated that 7\% (700) undergo additional tests that exclude the presence of heart disease, including 700 echocardiograms, 133 (19\%) exercise tests, 35 (5\%) Holter, and 7 (1\%) cardiac catheterization, electrophysiologic and/or CMR studies. In addition, 200 (2\% of the 10000 ) athletes undergo more tests (198 echocardiograms, 164 exercise tests, 6 Holter recordings and 10 cardiac catheterizations, electrophysiologic and/or CMR studies) and are eventually disqualified from sport participation.

(4) The mortality rate without screening is assumed to remain as high as in the pre-screening period (4 per 100000 athletes). ${ }^{3}$ In 
contrast, the mortality of screened athletes follows the eventfree survival curve published in the Italian study that gradually descends to 0.43 per 100000 athletes during the last 4 years of screening. Assuming 100000 athletes are evaluated every year, for 2200000 athlete-screening-tests performed over a prolonged period of 22 years, ${ }^{3}$ the number of athletes expected to die in the absence of screening is 88 and the number of athletes expected to die despite screening is 35 . The number of lives saved with 2200000 screening tests is 53 . It should be acknowledged, however, that this figure does not take into consideration the number of cardiac arrests successfully resuscitated.

(5) The number of tests to be performed in order to save one life include 41000 electrocardiograms, 3700 echocardiograms, 1200 exercise stress tests, 54 Holter and 71 cardiac catheterization, electrophysiologic studies and/or CMR tests.

Taking these assumptions and the cost of each test into consideration, the cost of saving one life will vary considerably by country. In the USA, where diagnostic testing for screening purposes is not commonly reimbursed, the PPE and additional testing should be computed singularly, using the price reported for Medicare reimbursement, which will raise the cost to $>10$ million US dollars to save one life. ${ }^{171}$

It should be noted, though, that cost-efficacy analyses heavily rely on a priori assumptions (Table 4). Factors such as the cost of each test, the prevalence of underlying cardiomyopathies, the estimated SCD risk conferred by each cardiac condition and the sensitivity and specificity of screening tests critically influence outcomes. Wheeler et al. also based their analyses in the Italian experience. ${ }^{3}$ The authors concluded that adding a single ECG to history and physical examination at participation entry would save 2.06 life-years per 1000 young athletes screened at a cost of $\$ 42000$ per life-year saved. ${ }^{174}$ Nevertheless, this analysis does not consider the annual reevaluation carried out in the Italian screening programme.

An assessment of the cost/efficacy ECG-based PPE in Italy was reported by Corrado et al. in 2013. ${ }^{177}$ Corrado and collaborators used the current medical costs in Italy and assumed a constant mortality rate for the whole study period at $0.4 / 100000$ and 3.6/100 000 for screened and non-screened athletes, respectively. With these premises in mind, each life saved would cost $\sim € 1000000$. Assuming that young competitive athletes saved from SCD will live at least an additional 20 year period, the cost per year of life saved can be estimated at $\sim € 50000$ per year.

While other authors estimated a similar cost per life-year saved, ${ }^{172}$ Schoenbaum et al. found a remarkably higher $\$ 68000$ per life-year saved in 14 year-old athletes. ${ }^{176}$ The overall cost of an screening strategy including clinical history, physical examination, and ECG yields a variable cost-efficacy ratio ranging from $\$ 76100^{174}$ to roughly $\$ 100000^{175}$ per life-year saved, including therapeutic management and follow-up.

\section{Consensus statements}

The present document represents the effort of an expert panel of scientists and professionals to offer a comprehensive and unbiased overview of the several and still debated issues surrounding the best 
strategies to minimize the risk of cardiac arrest/sudden death in the athletic population.

The goal of the panel was to formulate a consensus, which represents a reasonable balance between the availability, sensitivity, specificity, and feasibility for implementation of the diagnostic tests deemed to the timely identification of $\mathrm{CV}$ disease at risk in the athletic population.

The major limitation of the present document is the limited scientific evidence inherent to most of the debated issues. Due to the scarcity of scientific studies concerning the impact of regular sport activities on the clinical presentation and outcome of several CV diseases, the panel acknowledges the difficulties inherent in formulating advices regarding the best diagnostic work out and the risk stratification for most CV disease.

With these limitations in mind, we summarize here the agreement existing within the panel with regard to the following points:

\section{Aim and nature of the preparticipation evaluation}

The primary scope of the PPE is to identify cardiac diseases clinically silent that may be associated with cardiac arrest/sudden cardiac death in relation with exercise training and competitive sport participation.

However, the scope of the PPE is not merely limited to prevention of SCD, but extends to the identification and appropriate management of those $\mathrm{CV}$ conditions that may worsen because of intensive athletic training, as well as PPE may be the time for primary prevention of CV disease and improvement of CV health, through educational, nutritional, and treatment interventions. Therefore, PPE is a preventive medical programme justifiable on ethical, social, and medical ground.

\section{Protocol of the preparticipation evaluation}

The protocol of PPE including clinical history, physical examination, and 12-lead ECG demonstrates to have superior diagnostic capability than just clinical history and physical examination. There is compelling scientific evidence that the 12-lead ECG improves substantially the diagnostic power of PPE, mostly due the capability to identity arrhythmogenic conditions at risk (cardiomyopathies and channelopathies).

Available data suggests that routine echocardiography or other imaging modalities do not add substantial diagnostic power to the PPE as a mass screening technique and do not appear to be cost/effective. Therefore, at the moment the ECG-based PPE represents the most effective protocol to evaluate athletes (i.e. best clinical practice), although several limitations should be acknowledged.

Relevant to the diagnostic capability of the ECG-screening protocol is the issue of false positive ECGs and the challenge of appropriate interpretation of the ECG in trained athletes. The updated recommendations for interpretation of the athlete's ECG (Seattle criteria) represent a useful document to the scope.

The evidence derived from the Veneto experience suggests that PPE based on ECG, implemented as mass screening in the athletic population is associated with substantial reduction in CV mortality.

\section{Implementation of the preparticipation evaluation}

The implementation of a PPE is a complex issue, in which the availability of trained medical/paramedical personnel, but also adequate logistic, as well as a supporting legal and economic framework should be taken into account. Some of the controversies related to implementation of PPE (e.g. Italy vs. US positions) represent an expression of the different cultural, social, and legal framework existing in these countries, but also reflect dissimilar analyses of scientific evidence.

This panel believes that PPE should be considered and advised for individuals performing regular, intense exercise, after proper information of both its benefits and limitations. This panel believes that sport organizations, such as the International Olympic Committee and national and international federations, share the responsibility to properly inform elite and professional athletes of the benefits and limitations of the PPE and to advise PPE in professional athletes on the basis of perceived responsibility and public scrutiny.

It goes far beyond the scope of the document to suggest global national PPE programmes. General population screenings remain within the strict framework of the government of countries, according to the perceived cultural, social, and legal priorities and available economic resources. Indeed, different interpretation of scientific evidence and opposing cultural attitudes underlay the current discrepancies on mandatory PPE.

\section{Future directions}

PPE programmes still have plenty of room for improvement. Sudden cardiac death during physical activity needs to be better characterized to provide a better understanding of its impact. Future research should pave the way for a better identification of individuals at risk, which is not only accomplished by their better identification, but also more accurate risk stratification allowing a lower burden of sport disqualification. The design of better non-invasive techniques should aim at identifying those conditions that remain silent with conventional actual techniques, namely coronary artery abnormalities and subclinical CAD. Such improvements will likely have an impact on the perception of athletes and the society of PPE, will reduce costs and improve efficiency.

\section{Acknowledgment}

EHRA Scientific Committee: Prof. Gregory YH Lip (chair), Prof. Bulent Gorenek (co-chair), Prof. Christian Sticherling, Prof. Laurent Fauchier, Prof. A. Goette, Prof. Werner Jung, Prof. Marc A Vos, Dr Michele Brignole, Dr Christian Elsner, Prof. Gheorghe-Andrei Dan, Dr Francisco Marin, Prof. Giuseppe Boriani, Dr Deirdre Lane, Prof. Carina Blomstrom Lundqvist, Dr Irina Savelieva.

\section{References}

1. Kim JH, Malhotra R, Chiampas G, d'Hemecourt P, Troyanos C, Cianca J et al. Cardiac arrest during long-distance running races. N Engl J Med 2012;366:130-40.

2. Harmon KG, Asif IM, Maleszewski JJ, Owens DS, Prutkin JM, Salerno JC et al. Incidence, etiology, and comparative frequency of sudden cardiac death in NCAA athletes: a decade in review. Circulation 2015;132:10-19.

3. Corrado D, Basso C, Pavei A, Michieli P, Schiavon M, Thiene G. Trends in sudden cardiovascular death in young competitive athletes after implementation of a preparticipation screening program. JAMA 2006;296:1593-601. 
4. Marijon E, Tafflet M, Celermajer DS, Dumas F, Perier MC, Mustafic H et al. Sportsrelated sudden death in the general population. Circulation 2011;124:672-81.

5. Corrado D, Basso C, Rizzoli G, Schiavon M, Thiene G. Does sports activity enhance the risk of sudden death in adolescents and young adults? J Am Coll Cardiol 2003;42:1959-63.

6. Dunn TP, Pickham D, Aggarwal S, Saini D, Kumar N, Wheeler MT et al. Limitations of current $\mathrm{AHA}$ guidelines and proposal of new guidelines for the preparticipation examination of athletes. Clin / Sport Med 2015;25:472-7.

7. Pelliccia A, Fagard R, Bjornstad HH, Anastassakis A, Arbustini E, Assanelli D et al. Recommendations for competitive sports participation in athletes with cardiovascular disease: a consensus document from the Study Group of Sports Cardiology of the Working Group of Cardiac Rehabilitation and Exercise Physiology and the Working Group of Myocardial and Pericardial Diseases of the European Society of Cardiology. Eur Heart J 2005;26:1422-45.

8. Borjesson M, Urhausen A, Kouidi E, Dugmore D, Sharma S, Halle M et al. Cardiovascular evaluation of middle-aged/ senior individuals engaged in leisure-time sport activities: position stand from the sections of exercise physiology and sports cardiology of the European Association of Cardiovascular Prevention and Rehabilitation. Eur J Cardiovasc Prev Rehabil 2011;18:446-58.

9. Elliott PM, Anastasakis A, Borger MA, Borggrefe M, Cecchi F, Charron P et al. 2014 ESC Guidelines on diagnosis and management of hypertrophic cardiomyopathy: the Task Force for the Diagnosis and Management of Hypertrophic Cardiomyopathy of the European Society of Cardiology (ESC). Eur Heart J 2014;35:2733-79.

10. Priori SG, Blomstrom-Lundqvist C, Mazzanti A, Blom N, Borggrefe M, Camm J et al. 2015 ESC Guidelines for the management of patients with ventricular arrhythmias and the prevention of sudden cardiac death: The Task Force for the Management of Patients with Ventricular Arrhythmias and the Prevention of Sudden Cardiac Death of the European Society of Cardiology (ESC)Endorsed by: Association for European Paediatric and Congenital Cardiology (AEPC). Europace 2015;17:1601-87.

11. Priori SG, Wilde AA, Horie M, Cho Y, Behr ER, Berul C et al. Executive summary: HRS/EHRA/APHRS expert consensus statement on the diagnosis and management of patients with inherited primary arrhythmia syndromes. Europace 2013; 15:1389-406

12. Pelliccia A, Corrado D, Bjornstad HH, Panhuyzen-Goedkoop N, Urhausen A, Carre $\mathrm{F}$ et al. Recommendations for participation in competitive sport and leisuretime physical activity in individuals with cardiomyopathies, myocarditis and pericarditis. Eur J Cardiovasc Prev Rehabil 2006;13:876-85.

13. Perk J, De Backer G, Gohlke H, Graham I, Reiner Z, Verschuren M et al. The Fifth Joint Task Force of the European Society of Cardiology and Other Societies on Cardiovascular Disease Prevention in Clinical Practice (constituted by representatives of nine societies and by invited experts). Eur Heart J 2012;33:1635-701.

14. Harmon KG, Drezner JA, Wilson MG, Sharma S. Incidence of sudden cardiac death in athletes: a state-of-the-art review. Heart 2014;100:1227-34.

15. Toresdahl BG, Rao AL, Harmon KG, Drezner JA. Incidence of sudden cardiac arrest in high school student athletes on school campus. Heart Rhythm 2014;11: 1190-4.

16. Risgaard B, Winkel BG, Jabbari R, Glinge C, Ingemann-Hansen $O$, Thomsen JL et al. Sports-related sudden cardiac death in a competitive and a noncompetitive athlete population aged 12 to 49 years: data from an unselected nationwide study in Denmark. Heart Rhythm 2014;11:1673-81.

17. Maron BJ, Haas TS, Murphy CJ, Ahluwalia A, Rutten-Ramos S. Incidence and causes of sudden death in U.S. college athletes. Am Coll Cardiol 2014;63:1636-43.

18. Corrado D, Thiene G, Nava A, Rossi L, Pennelli N. Sudden death in young competitive athletes: clinicopathologic correlations in 22 cases. Am J Med 1990;89: 588-96.

19. Maron BJ, Doerer JJ, Haas TS, Tierney DM, Mueller FO. Sudden deaths in young competitive athletes: analysis of 1866 deaths in the United States, 1980-2006. Circulation 2009;119:1085-92.

20. Eckart RE, Shry EA, Burke AP, McNear JA, Appel DA, Castillo-Rojas LM et al. Sudden death in young adults: an autopsy-based series of a population undergoing active surveillance. J Am Coll Cardiol 2011;58:1254-61.

21. Holst AG, Winkel BG, Theilade J, Kristensen IB, Thomsen JL, Ottesen GL et al. Incidence and etiology of sports-related sudden cardiac death in Denmarkimplications for preparticipation screening. Heart Rhythm 2010;7:1365-71.

22. Solberg EE, Borjesson M, Sharma S, Papadakis M, Wilhelm M, Drezner JA et al. Sudden cardiac arrest in sports-need for uniform registration: A Position Paper from the Sport Cardiology Section of the European Association for Cardiovascular Prevention and Rehabilitation. Eur J Prev Cardiol 2016;23:657-67.

23. Maron BJ, Shirani J, Poliac L, Mathenge R, Roberts WC, Mueller FO. Sudden death in young competitive athletes: clinical, demographic, and pathological profiles. JAMA 1996;276:199-204.

24. Suarez-Mier MP, Aguilera B. Causes of sudden death during sports activities in Spain. Rev Esp Cardiol 2002;55:347-58.
25. Watkins H, Thierfelder L, Hwang DS, McKenna W, Seidman JG, Seidman CE. Sporadic hypertrophic cardiomyopathy due to de novo myosin mutations. J Clin Invest 1992;90:1666-71.

26. Semsarian C, Ingles J, Maron MS, Maron BJ. New perspectives on the prevalence of hypertrophic cardiomyopathy. J Am Coll Cardiol 2015;65:1249-54.

27. Maron BJ, Niimura H, Casey SA, Soper MK, Wright GB, Seidman JG et al. Development of left ventricular hypertrophy in adults in hypertrophic cardiomyopathy caused by cardiac myosin-binding protein $\mathrm{C}$ gene mutations. J Am Coll Cardiol 2001;38:315-21.

28. O'Mahony C, Jichi F, Pavlou M, Monserrat L, Anastasakis A, Rapezzi C et al. A novel clinical risk prediction model for sudden cardiac death in hypertrophic cardiomyopathy (HCM risk-SCD). Eur Heart J 2014;35:2010-20.

29. Maron BJ, Pelliccia A. The heart of trained athletes: cardiac remodeling and the risks of sports, including sudden death. Circulation 2006;114:1633-44.

30. Caselli S, Maron MS, Urbano-Moral JA, Pandian NG, Maron BJ, Pelliccia A. Differentiating left ventricular hypertrophy in athletes from that in patients with hypertrophic cardiomyopathy. Am J Cardiol 2014;114:1383-9.

31. Sheikh N, Papadakis M, Schnell F, Panoulas V, Malhotra A, Wilson M et al. Clinical profile of athletes with hypertrophic cardiomyopathy. Circ Cardiovasc Imaging 2015;8:e003454.

32. Ryan MP, Cleland JG, French JA, Joshi J, Choudhury L, Chojnowska L et al. The standard electrocardiogram as a screening test for hypertrophic cardiomyopathy. Am J Cardiol 1995;76:689-94.

33. Charron P, Dubourg O, Desnos M, Bouhour JB, Isnard R, Hagege A et al. Diagnostic value of electrocardiography and echocardiography for familial hypertrophic cardiomyopathy in genotyped children. Eur Heart J 1998;19:1377-82.

34. Pelliccia A, Maron BJ, Culasso F, Di Paolo FM, Spataro A, Biffi A et al. Clinical significance of abnormal electrocardiographic patterns in trained athletes. Circulation 2000;102:278-84.

35. Papadakis M, Carre F, Kervio G, Rawlins J, Panoulas VF, Chandra $\mathrm{N}$ et al. The prevalence, distribution, and clinical outcomes of electrocardiographic repolarization patterns in male athletes of African/Afro-Caribbean origin. Eur Heart J 2011;32:2304-13.

36. Sheikh N, Papadakis M, Ghani S, Zaidi A, Gati S, Adami PE et al. Comparison of electrocardiographic criteria for the detection of cardiac abnormalities in elite black and white athletes. Circulation 2014;129:1637-49.

37. Corrado D, Basso C, Thiene G, McKenna WJ, Davies MJ, Fontaliran F et al. Spectrum of clinicopathologic manifestations of arrhythmogenic right ventricular cardiomyopathy/dysplasia: a multicenter study. J Am Coll Cardiol 1997;30: $1512-20$.

38. Corrado D, Basso C, Thiene G. Arrhythmogenic right ventricular cardiomyopathy: diagnosis, prognosis, and treatment. Heart 2000;83:588-95.

39. Corrado D, Thiene G. Arrhythmogenic right ventricular cardiomyopathy/dysplasia: clinical impact of molecular genetic studies. Circulation 2006;113:1634-7.

40. Corrado D, Basso C, Pilichou K, Thiene G. Molecular biology and clinical management of arrhythmogenic right ventricular cardiomyopathy/dysplasia. Heart 2011; 97:530-9.

41. Migliore F, Zorzi A, Silvano M, Bevilacqua M, Leoni L, Marra MP et al. Prognostic value of endocardial voltage mapping in patients with arrhythmogenic right ventricular cardiomyopathy/dysplasia. Circ Arrhythm Electrophysiol 2013;6:167-76.

42. James CA, Bhonsale A, Tichnell C, Murray B, Russell SD, Tandri $\mathrm{H}$ et al. Exercise increases age-related penetrance and arrhythmic risk in arrhythmogenic right ventricular dysplasia/cardiomyopathy-associated desmosomal mutation carriers. I Am Coll Cardiol 2013;62:1290-7.

43. Saberniak J, Hasselberg NE, Borgquist R, Platonov PG, Sarvari SI, Smith HJ et al. Vigorous physical activity impairs myocardial function in patients with arrhythmogenic right ventricular cardiomyopathy and in mutation positive family members. Eur J Heart Fail 2014;16:1337-44

44. Ruwald AC, Marcus F, Estes NA 3rd, Link M, McNitt S, Polonsky B et al. Association of competitive and recreational sport participation with cardiac events in patients with arrhythmogenic right ventricular cardiomyopathy: results from the North American multidisciplinary study of arrhythmogenic right ventricular cardiomyopathy. Eur Heart J 2015;36:1735-43.

45. McKenna WJ, Thiene G, Nava A, Fontaliran F, Blomstrom-Lundqvist C Fontaine $\mathrm{G}$ et al. Diagnosis of arrhythmogenic right ventricular dysplasia/cardiomyopathy. Task Force of the Working Group Myocardial and Pericardial Disease of the European Society of Cardiology and of the Scientific Council on Cardiomyopathies of the International Society and Federation of Cardiology. Br Heart J 1994; 71:215-8.

46. Marcus FI, McKenna WJ, Sherrill D, Basso C, Bauce B, Bluemke DA et al. Diagnosis of arrhythmogenic right ventricular cardiomyopathy/dysplasia: proposed modification of the Task Force Criteria. Eur Heart J 2010;31:806-14.

47. Corrado D, Basso C, Thiene G. Arrhythmogenic right ventricular cardiomyopathy: an update. Heart 2009;95:766-73. 
48. Corrado D, Schmied C, Basso C, Borjesson M, Schiavon M, Pelliccia A et al. Risk of sports: do we need a pre-participation screening for competitive and leisure athletes? Eur Heart J 2011;32:934-44.

49. Platonov PG, Calkins H, Hauer RN, Corrado D, Svendsen JH, Wichter T et al. High interobserver variability in the assessment of epsilon waves: implications for diagnosis of arrhythmogenic right ventricular cardiomyopathy/dysplasia. Heart Rhythm 2016;13:208-16.

50. Marra MP, Leoni L, Bauce B, Corbetti F, Zorzi A, Migliore F et al. Imaging study of ventricular scar in arrhythmogenic right ventricular cardiomyopathy: comparison of 3D standard electroanatomical voltage mapping and contrast-enhanced cardiac magnetic resonance. Circ Arrhythm Electrophysiol 2012;5:91-100.

51. Gomes J, Finlay M, Ahmed AK, Ciaccio EJ, Asimaki A, Saffitz JE et al. Electrophysiological abnormalities precede overt structural changes in arrhythmogenic right ventricular cardiomyopathy due to mutations in desmoplakin-A combined murine and human study. Eur Heart J 2012;33:1942-53.

52. Te Rijdt WP, Jongbloed JD, de Boer RA, Thiene G, Basso C, van den Berg MP et al. Clinical utility gene card for: arrhythmogenic right ventricular cardiomyopathy (ARVC). Eur J Hum Genet 2014;22, doi:10.1038/ejhg.2013.124.

53. Angelini P. Coronary artery anomalies: an entity in search of an identity. Circulation 2007;115:1296-305.

54. Basso C, Maron BJ, Corrado D, Thiene G. Clinical profile of congenital coronary artery anomalies with origin from the wrong aortic sinus leading to sudden death in young competitive athletes. J Am Coll Cardiol 2000;35:1493-501.

55. Wyman RA, Chiu RY, Rahko PS. The 5-minute screening echocardiogram for athletes. J Am Soc Echocardiogr 2008;21:786-8.

56. Hiss RG, Lamb LE. Electrocardiographic findings in 122,043 individuals. Circulation 1962;25:947-61.

57. Aras D, Tufekcioglu O, Ergun K, Ozeke O, Yildiz A, Topaloglu S et al. Clinical features of isolated ventricular noncompaction in adults long-term clinical course, echocardiographic properties, and predictors of left ventricular failure. J Card Fail 2006;12:726-33.

58. Towbin JA, Lorts A, Jefferies JL. Left ventricular non-compaction cardiomyopathy. Lancet 2015;386:813-25.

59. Brescia ST, Rossano JW, Pignatelli R, Jefferies JL, Price JF, Decker JA et al. Mortality and sudden death in pediatric left ventricular noncompaction in a tertiary referral center. Circulation 2013;127:2202-8.

60. Caselli S, Attenhofer Jost CH, Jenni R, Pelliccia A. Left ventricular noncompaction diagnosis and management relevant to pre-participation screening of athletes. Am J Cardiol 2015;116:801-8.

61. Gati S, Chandra N, Bennett RL, Reed M, Kervio G, Panoulas VF et al. Increased left ventricular trabeculation in highly trained athletes: do we need more stringent criteria for the diagnosis of left ventricular non-compaction in athletes? Heart 2013; 99:401-8

62. Pinto YM, Elliott PM, Arbustini E, Adler Y, Anastasakis A, Bohm M et al. Proposal for a revised definition of dilated cardiomyopathy, hypokinetic non-dilated cardiomyopathy, and its implications for clinical practice: a position statement of the ESC working group on myocardial and pericardial diseases. Eur Heart / 2016;37: 1850-8.

63. Marijon E, Uy-Evanado A, Reinier K, Teodorescu C, Narayanan K, Jouven X et al. Sudden cardiac arrest during sports activity in middle age. Circulation 2015;131: 1384-91.

64. Pelliccia A, Culasso F, Di Paolo FM, Maron BJ. Physiologic left ventricular cavity dilatation in elite athletes. Ann Intern Med 1999;130:23-31.

65. Siu SC, Silversides CK. Bicuspid aortic valve disease. J Am Coll Cardiol 2010;55: 2789-00.

66. Lieve KV, Wilde AA. Inherited ion channel diseases: a brief review. Europace 2015; 17(Suppl 2):ii1-6.

67. Schwartz PJ, Stramba-Badiale M, Crotti L, Pedrazzini M, Besana A, Bosi G et al. Prevalence of the congenital long-QT syndrome. Circulation 2009;120:1761-7.

68. Nakano Y, Shimizu W. Genetics of long-QT syndrome. J Hum Genet 2016;61: $51-5$.

69. Ackerman MJ, Priori SG, Willems S, Berul C, Brugada R, Calkins $\mathrm{H}$ et al. HRS/ EHRA expert consensus statement on the state of genetic testing for the channelopathies and cardiomyopathies. Europace 2011;13:1077-109.

70. Fukuyama M, Wang Q, Kato K, Ohno S, Ding WG, Toyoda F et al. Long QT syndrome type 8: novel CACNA1C mutations causing QT prolongation and variant phenotypes. Europace 2014;16:1828-37.

71. Nguyen HL, Pieper GH, Wilders R. Andersen-Tawil syndrome: clinical and molecular aspects. Int J Cardiol 2013;170:1-16.

72. Splawski I, Timothy KW, Sharpe LM, Decher N, Kumar P, Bloise R et al. Ca(V)1.2 calcium channel dysfunction causes a multisystem disorder including arrhythmia and autism. Cell 2004;119:19-31.

73. Schwartz PJ, Priori SG, Spazzolini C, Moss AJ, Vincent GM, Napolitano C et al. Genotype-phenotype correlation in the long-QT syndrome: gene-specific triggers for life-threatening arrhythmias. Circulation 2001;103:89-95.
74. Schwartz PJ, Moss AJ, Vincent GM, Crampton RS. Diagnostic criteria for the long QT syndrome. An update. Circulation 1993;88:782-4.

75. Vincent GM, Jaiswal D, Timothy KW. Effects of exercise on heart rate, QT, QTc and QT/QS2 in the Romano-Ward inherited long QT syndrome. Am / Cardiol 1991;68:498-503.

76. Swan H, Viitasalo M, Piippo K, Laitinen P, Kontula K, Toivonen L. Sinus node function and ventricular repolarization during exercise stress test in long QT syndrome patients with KvLQT1 and HERG potassium channel defects. J Am Coll Cardiol 1999;34:823-9.

77. Horner JM, Horner MM, Ackerman MJ. The diagnostic utility of recovery phase QTc during treadmill exercise stress testing in the evaluation of long QT syndrome. Heart Rhythm 2011;8:1698-704.

78. Schwartz PJ, Crotti L. QTc behavior during exercise and genetic testing for the long-QT syndrome. Circulation 2011;124:2181-4.

79. Sy RW, van der Werf C, Chattha IS, Chockalingam P, Adler A, Healey JS et al. Derivation and validation of a simple exercise-based algorithm for prediction of genetic testing in relatives of LQTS probands. Circulation 2011;124:2187-94.

80. Viskin S, Postema PG, Bhuiyan ZA, Rosso R, Kalman JM, Vohra JK et al. The response of the QT interval to the brief tachycardia provoked by standing: a bedside test for diagnosing long QT syndrome. J Am Coll Cardiol 2010;55:1955-61.

81. Adler A, van der Werf C, Postema PG, Rosso R, Bhuiyan ZA, Kalman JM et al. The phenomenon of 'QT stunning': the abnormal QT prolongation provoked by standing persists even as the heart rate returns to normal in patients with long QT syndrome. Heart Rhythm 2012;9:901-8.

82. Stramba-Badiale M, Priori SG, Napolitano C, Locati EH, Vinolas X, Haverkamp W et al. Gene-specific differences in the circadian variation of ventricular repolarization in the long QT syndrome: a key to sudden death during sleep? Ital Heart J 2000;1:323-8.

83. Priori SG, Schwartz PJ, Napolitano C, Bloise R, Ronchetti E, Grillo M et al. Risk stratification in the long-QT syndrome. N Engl J Med 2003;348:1866-74.

84. Guerrier K, Kwiatkowski D, Czosek RJ, Spar DS, Anderson JB, Knilans TK. Short QT interval prevalence and clinical outcomes in a pediatric population. Circ Arrhythm Electrophysiol 2015;8:1460-4.

85. Anttonen O, Junttila MJ, Rissanen H, Reunanen A, Viitasalo M, Huikuri HV. Prevalence and prognostic significance of short QT interval in a middle-aged Finnish population. Circulation 2007;116:714-20.

86. Dhutia H, Malhotra A, Parpia S, Gabus V, Finocchiaro G, Mellor G et al. The prevalence and significance of a short QT interval in 18825 low-risk individuals including athletes. BrJ Sports Med 2016;50:124-9.

87. Priori SG, Napolitano C, Memmi M, Colombi B, Drago F, Gasparini M et al. Clinical and molecular characterization of patients with catecholaminergic polymorphic ventricular tachycardia. Circulation 2002;106:69-74.

88. Marjamaa A, Hiippala A, Arrhenius B, Lahtinen AM, Kontula K, Toivonen L et al. Intravenous epinephrine infusion test in diagnosis of catecholaminergic polymorphic ventricular tachycardia. J Cardiovasc Electrophysiol 2012;23:194-9.

89. van $\operatorname{der}$ Werf $\mathrm{C}$, Z winderman AH, Wilde AA. Therapeutic approach for patients with catecholaminergic polymorphic ventricular tachycardia: state of the art and future developments. Europace 2012;14:175-83.

90. Brugada P, Brugada J. Right bundle branch block, persistent ST segment elevation and sudden cardiac death: a distinct clinical and electrocardiographic syndrome. A multicenter report. I Am Coll Cardiol 1992;20:1391-6.

91. Catalano O, Antonaci S, Moro G, Mussida M, Frascaroli M, Baldi M et al. Magnetic resonance investigations in Brugada syndrome reveal unexpectedly high rate of structural abnormalities. Eur Heart J 2009;30:2241-8.

92. Schulze-Bahr E, Eckardt L, Breithardt G, Seidl K, Wichter T, Wolpert C et al. Sodium channel gene (SCN5A) mutations in 44 index patients with Brugada syndrome: different incidences in familial and sporadic disease. Hum Mutat 2003 21:651-2.

93. Rudic B, Schimpf R, Veltmann C, Doesch C, Tulumen E, Schoenberg SO et al. Brugada syndrome: clinical presentation and genotype-correlation with magnetic resonance imaging parameters. Europace 2015. In press. doi:10.1093/europace/ euv300.

94. Mizusawa Y, Wilde AA. Brugada syndrome. Circ Arrhythm Electrophysiol 2012;5 606-16.

95. Amin AS, de Groot EA, Ruijter JM, Wilde AA, Tan HL. Exercise-induced ECG changes in Brugada syndrome. Circ Arrhythm Electrophysiol 2009;2:531-9.

96. Makimoto H, Nakagawa E, Takaki H, Yamada Y, Okamura H, Noda T et al. Augmented ST-segment elevation during recovery from exercise predicts cardiac events in patients with Brugada syndrome. J Am Coll Cardiol 2010;56: 1576-84.

97. Thompson PD, Franklin BA, Balady GJ, Blair SN, Corrado D, Estes NA III et al. Exercise and acute cardiovascular events placing the risks into perspective: a scientific statement from the American Heart Association Council on Nutrition, Physical Activity, and Metabolism and the Council on Clinical Cardiology. Circulation 2007:115:2358-68. 
98. Giri S, Thompson PD, Kiernan FJ, Clive J, Fram DB, Mitchel JF et al. Clinical and angiographic characteristics of exertion-related acute myocardial infarction. JAMA 1999;282:1731-6.

99. Fletcher GF, Ades PA, Kligfield P, Arena R, Balady G], Bittner VA et al. Exercise standards for testing and training: a scientific statement from the American Heart Association. Circulation 2013;128:873-934.

100. Guerri-Guttenberg RA, Grana DR, Ambrosio G, Milei J. Chagas cardiomyopathy: Europe is not spared! Eur Heart J 2008;29:2587-91.

101. Rassi A Jr, Rassi A, Marin-Neto JA. Chagas disease. Lancet 2010;375:1388-402.

102. Dias JC. The indeterminate form of human chronic Chagas' disease A clinical epidemiological review. Rev Soc Bras Med Trop 1989;22:147-56.

103. Nunes MC, Dones W, Morillo CA, Encina JJ, Ribeiro AL, Council on Chagas Disease of the Interamerican Society of Cardiology. Chagas disease: an overview of clinical and epidemiological aspects. J Am Coll Cardiol 2013;62: 767-76.

104. Sternick EB, Martinelli M, Sampaio R, Gerken LM, Teixeira RA, Scarpelli R et al. Sudden cardiac death in patients with chagas heart disease and preserved left ventricular function. J Cardiovasc Electrophysiol 2006;17:113-6.

105. de Paola AA, Gomes JA, Terzian AB, Miyamoto MH, Martinez Fo EE. Ventricular tachycardia during exercise testing as a predictor of sudden death in patients with chronic chagasic cardiomyopathy and ventricular arrhythmias. Br Heart J 1995;74: 293-5.

106. Martinelli Filho M, Sosa E, Nishioka S, Scanavacca M, Bellotti G, Pileggi F. Clinical and electrophysiologic features of syncope in chronic chagasic heart disease. J Cardiovasc Electrophysiol 1994;5:563-70.

107. Shivkumar K, Boyle NG, Scanavacca M, Sosa E. Epicardial ablation of ventricular tachycardia in Chagas heart disease. In: Shivkumar K, Boyle NG (eds). Cardiac Electrophysiology Clinics: Epicardial Interventions in Electrophysiology. Philadelphia: Saunders; 2010, p55-67.

108. Matos LD, Azevedo LF, Brum PC, Sosa EA, Martinelli M, Negrao CE. Long distance runner with dilated cardiomyopathy and excellent performance. Arq Bras Cardiol 2011;96:e3-6.

109. Cruz FM, Sanz-Rosa D, Roche-Molina M, Garcia-Prieto J, Garcia-Ruiz JM, Pizarro G et al. Exercise triggers ARVC phenotype in mice expressing a diseasecausing mutated version of human plakophilin-2. J Am Coll Cardiol 2015;65: 1438-50.

110. Kirchhof P, Fabritz L, Zwiener M, Witt H, Schafers M, Zellerhoff S et al. Age- and training-dependent development of arrhythmogenic right ventricular cardiomyopathy in heterozygous plakoglobin-deficient mice. Circulation 2006;114: 1799-806.

111. Konhilas JP, Watson PA, Maass A, Boucek DM, Horn T, Stauffer BL et al. Exercise can prevent and reverse the severity of hypertrophic cardiomyopathy. Circ Res 2006;98:540-8.

112. Heidbuchel H, Hoogsteen J, Fagard R, Vanhees L, Ector H, Willems R et al. High prevalence of right ventricular involvement in endurance athletes with ventricular arrhythmias. Role of an electrophysiologic study in risk stratification. Eur Heart J 2003;24:1473-80.

113. La Gerche A, Robberecht C, Kuiperi C, Nuyens D, Willems R, de Ravel T et al. Lower than expected desmosomal gene mutation prevalence in endurance athletes with complex ventricular arrhythmias of right ventricular origin. Heart 2010;96:1268-74.

114. Benito B, Gay-Jordi G, Serrano-Mollar A, Guasch E, Shi Y, Tardif JC et al. Cardiac arrhythmogenic remodeling in a rat model of long-term intensive exercise training. Circulation 2011;123:13-22.

115. Sawant AC, Bhonsale A, Te Riele AS, Tichnell C, Murray B, Russell SD et al. Exercise has a disproportionate role in the pathogenesis of arrhythmogenic right ventricular dysplasia/cardiomyopathy in patients without desmosomal mutations. J Am Heart Assoc 2014;3:e001471.

116. La Gerche A, Heidbuchel H. Can intensive exercise harm the heart? You can get too much of a good thing. Circulation 2014;130:992-1002.

117. Heidbuchel H, Prior DL, La Gerche A. Ventricular arrhythmias associated with long-term endurance sports: what is the evidence? Br J Sports Med 2012; 46(Suppl 1):i44-50.

118. Abergel E, Chatellier G, Hagege AA, Oblak A, Linhart A, Ducardonnet A et al. Serial left ventricular adaptations in world-class professional cyclists: implications for disease screening and follow-up. J Am Coll Cardiol 2004;44:144-9.

119. Kiel RJ, Smith FE, Chason J, Khatib R, Reyes MP. Coxsackievirus B3 myocarditis in $\mathrm{C} 3 \mathrm{H} / \mathrm{HeJ}$ mice: description of an inbred model and the effect of exercise on virulence. Eur J Epidemiol 1989;5:348-50

120. Schnell F, Claessen G, La Gerche A, Bogaert J, Lentz PA, Claus P et al. Subepicardial delayed gadolinium enhancement in asymptomatic athletes: let sleeping dogs lie? Br J Sports Med 2016;50:111-7.

121. Corrado D, Basso C, Schiavon M, Thiene G. Screening for hypertrophic cardiomyopathy in young athletes. N Engl J Med 1998;339:364-9.
122. Harmon KG, Zigman M, Drezner JA. The effectiveness of screening history, physical exam, and ECG to detect potentially lethal cardiac disorders in athletes: a systematic review/meta-analysis. J Electrocardiol 2015;48:329-38.

123. Brosnan M, La Gerche A, Kalman J, Lo W, Fallon K, Maclsaac A et al. Comparison of frequency of significant electrocardiographic abnormalities in endurance versus nonendurance athletes. Am J Cardiol 2014;113:1567-73.

124. Senturk T, Xu H, Puppala K, Krishnan B, Sakaguchi S, Chen LY et al. Cardiac pauses in competitive athletes: a systematic review examining the basis of current practice recommendations. Europace 2015. In press. doi:10.1093/europace/euv373.

125. Brosnan M, La Gerche A, Kalman J, Lo W, Fallon K, Maclsaac A et al. The Seattle Criteria increase the specificity of preparticipation ECG screening among elite athletes. Br J Sports Med 2014;48:1144-50.

126. Rowin EJ, Maron BJ, Appelbaum E, Link MS, Gibson CM, Lesser JR et al. Significance of false negative electrocardiograms in preparticipation screening of athletes for hypertrophic cardiomyopathy. Am J Cardiol 2012;110:1027-32.

127. Drezner JA, Fudge J, Harmon KG, Berger S, Campbell RM, Vetter VL. Warning symptoms and family history in children and young adults with sudden cardiac arrest. J Am Board Fam Med 2012;25:408-15.

128. Maron BJ, Friedman RA, Kligfield P, Levine BD, Viskin S, Chaitman BR et al. Assessment of the 12-lead ECG as a screening test for detection of cardiovascular disease in healthy general populations of young people (12-25 years of age): a scientific statement from the American Heart Association and the American College of Cardiology. Circulation 2014;130:1303-34.

129. Corrado D, Pelliccia A, Heidbuchel H, Sharma S, Link M, Basso $C$ et al. Recommendations for interpretation of 12-lead electrocardiogram in the athlete. Eur Heart J 2010;31:243-59.

130. Drezner JA, Ackerman MJ, Anderson J, Ashley E, Asplund CA, Baggish AL et al. Electrocardiographic interpretation in athletes: the 'Seattle Criteria'. Br J Sports Med 2013;47:122-4.

131. Pickham D, Zarafshar S, Sani D, Kumar N, Froelicher V. Comparison of three ECG criteria for athlete pre-participation screening. J Electrocardiol 2014;47:769-74.

132. Berge HM, Gjesdal K, Andersen TE, Solberg EE, Steine K. Prevalence of abnormal ECGs in male soccer players decreases with the Seattle criteria, but is still high. Scand J Med Sci Sports 2015;25:501-8.

133. Corrado D, Calore C, Zorzi A, Migliore F. Improving the interpretation of the athlete's electrocardiogram. Eur Heart J 2013;34:3606-9.

134. Viskin S, Rosovski U, Sands AJ, Chen E, Kistler PM, Kalman JM et al. Inaccurate electrocardiographic interpretation of long QT: the majority of physicians cannot recognize a long QT when they see one. Heart Rhythm 2005;2:569-74.

135. Berte B, Duytschaever M, Elices J, Kataria V, Timmers L, Van Heuverswyn F et al. Variability in interpretation of the electrocardiogram in young athletes: an unrecognized obstacle for electrocardiogram-based screening protocols. Europace 2015;17:1435-40.

136. Magalski A, Maron BJ, Main ML, McCoy M, Florez A, Reid KJ et al. Relation of race to electrocardiographic patterns in elite American football players. J Am Coll Cardiol 2008;51:2250-5.

137. Glover DW, Glover DW, Maron BJ. Evolution in the process of screening United States high school student-athletes for cardiovascular disease. Am J Cardiol 2007; 100:1709-12.

138. Heidbuchel H, Papadakis M, Panhuyzen-Goedkoop N, Carre F, Dugmore D, Mellwig KP et al. Position paper: proposal for a core curriculum for a European Sports Cardiology qualification. Eur J Prev Cardiol 2013;20:889-903.

139. Papadakis M, Chandra N, Sharma S. Controversies relating to preparticipation cardiovascular screening in young athletes: time for a realistic solution? $\mathrm{Br}$ J Sports Med 2011:45:165-6.

140. Solberg EE, Bjornstad TH, Andersen TE, Ekeberg O. Cardiovascular preparticipation screening does not distress professional football players. Eur J Prev Cardiol 2012;19:571-7.

141. Asif IM, Johnson S, Schmieg J, Smith T, Rao AL, Harmon KG et al. The psychological impact of cardiovascular screening: the athlete's perspective. Br J Sports Med 2014;48:1162-6.

142. Sharma S, Estes NA III, Vetter VL, Corrado D. Clinical decisions. Cardiac screening before participation in sports. N Engl J Med 2013;369:2049-53.

143. Tasaki H, Hamasaki Y, Ichimaru T. Mass screening for heart disease of school children in Saga city: 7-year follow up study. Jpn Circ J 1987;51:1415-20.

144. Vetter VL, Dugan N, Guo R, Mercer-Rosa L, Gleason M, Cohen M et al. A pilot study of the feasibility of heart screening for sudden cardiac arrest in healthy children. Am Heart J 2011;161:1000-1006.e3.

145. Lahav D, Leshno M, Brezis M. Is an exercise tolerance test indicated before beginning regular exercise? A decision analysis. J Gen Intern Med 2009;24:934-8.

146. Gianrossi R, Detrano R, Mulvihill D, Lehmann K, Dubach P, Colombo A et al. Exercise-induced ST depression in the diagnosis of coronary artery disease. A meta-analysis. Circulation 1989;80:87-98. 
147. Schnell F, Riding N, O'Hanlon R, Axel Lentz P, Donal E, Kervio G et al. Recognition and significance of pathological T-wave inversions in athletes. Circulation 2015;131: 165-73.

148. Feinstein RA, Colvin E, Kimoh M. Echocardiographic screening as part of a preparticipation examination. Clin J Sport Med 1993;3:149-52.

149. Weidenbener EJ, Krauss MD, Waller BF, Taliercio CP. Incorporation of screening echocardiography in the preparticipation exam. Clin J Sport Med 1995;5:86-9.

150. Anderson JB, Grenier M, Edwards NM, Madsen NL, Czosek RJ, Spar DS et al. Usefulness of combined history, physical examination, electrocardiogram, and limited echocardiogram in screening adolescent athletes for risk for sudden cardiac death. Am J Cardiol 2014;114:1763-7.

151. Riding NR, Sharma S, Salah O, Khalil N, Carre F, George KP et al. Systematic echocardiography is not efficacious when screening an ethnically diverse cohort of athletes in West Asia. Eur J Prev Cardiol 2015;22:263-70.

152. Lewis JF, Maron BJ, Diggs JA, Spencer JE, Mehrotra PP, Curry CL. Preparticipation echocardiographic screening for cardiovascular disease in a large, predominantly black population of collegiate athletes. Am J Cardiol 1989;64:1029-33.

153. Zaidi A, Ghani S, Sharma R, Oxborough D, Panoulas VF, Sheikh $N$ et al. Physiological right ventricular adaptation in elite athletes of African and Afro-Caribbean origin. Circulation 2013;127:1783-92.

154. Jellis C, Martin J, Narula J, Marwick TH. Assessment of nonischemic myocardial fibrosis. I Am Coll Cardiol 2010;56:89-97.

155. La Gerche A, Claessen G, Dymarkowski S, Voigt JU, De Buck F, Vanhees L et al. Exercise-induced right ventricular dysfunction is associated with ventricular arrhythmias in endurance athletes. Eur Heart J 2015;36:1998-2010.

156. La Gerche A, Burns AT, Mooney DJ, Inder WJ, Taylor AJ, Bogaert I et al. Exercise-induced right ventricular dysfunction and structural remodelling in endurance athletes. Eur Heart J 2012;33:998-1006.

157. Wilson M, O'Hanlon R, Prasad S, Deighan A, Macmillan P, Oxborough D et al. Diverse patterns of myocardial fibrosis in lifelong, veteran endurance athletes. J Appl Physiol 2011;110:1622-6.

158. Davis JA, Cecchin F, Jones TK, Portman MA. Major coronary artery anomalies in a pediatric population: incidence and clinical importance. J Am Coll Cardiol 2001;37: 593-7.

159. Albert CM, Mittleman MA, Chae CU, Lee IM, Hennekens CH, Manson JE. Triggering of sudden death from cardiac causes by vigorous exertion. N Engl J Med 2000; 343:1355-61.

160. Yeboah J, McClelland RL, Polonsky TS, Burke GL, Sibley CT, O'Leary D et al. Comparison of novel risk markers for improvement in cardiovascular risk assessment in intermediate-risk individuals. JAMA 2012;308:788-95.

161. Mohlenkamp S, Lehmann N, Breuckmann F, Brocker-Preuss M, Nassenstein K, Halle $M$ et al. Running: the risk of coronary events: prevalence and prognostic relevance of coronary atherosclerosis in marathon runners. Eur Heart J 2008;29: 1903-10.

162. Schnohr P, O'Keefe JH, Marott JL, Lange P, Jensen GB. Dose of jogging and longterm mortality: the Copenhagen City Heart Study. J Am Coll Cardiol 2015;65: 411-9.

163. Maron BJ, Levine BD, Washington RL, Baggish AL, Kovacs RJ, Maron MS. Eligibility and disqualification recommendations for competitive athletes with cardiovascular abnormalities: Task Force 2: preparticipation screening for cardiovascular disease in competitive athletes: a scientific statement from the American Heart
Association and American College of Cardiology. J Am Coll Cardiol 2015;66: 2356-61.

164. Dvorak J, Grimm K, Schmied C, Junge A. Development and implementation of a standardized precompetition medical assessment of international elite football players - 2006 FIFA World Cup Germany. Clin J Sport Med 2009;19:316-21.

165. Ljungqvist A, Jenoure $P$, Engebretsen L, Alonso JM, Bahr R, Clough $A$ et al. The International Olympic Committee (IOC) Consensus Statement on periodic health evaluation of elite athletes March 2009. Br J Sports Med 2009;43:631-43.

166. Viskin S. Antagonist: routine screening of all athletes prior to participation in competitive sports should be mandatory to prevent sudden cardiac death. Heart Rhythm 2007;4:525-8.

167. Steinvil A, Chundadze T, Zeltser D, Rogowski O, Halkin A, Galily Y et al. Mandatory electrocardiographic screening of athletes to reduce their risk for sudden death proven fact or wishful thinking? J Am Coll Cardiol 2011;57:1291-6.

168. Suissa S. Immortal time bias in pharmaco-epidemiology. Am J Epidemiol 2008;167: 492-9.

169. Roberts WO, Maron BJ. Evidence for decreasing occurrence of sudden cardiac death associated with the marathon. J Am Coll Cardiol 2005;46:1373-4.

170. Maron BJ, Haas TS, Doerer JJ, Thompson PD, Hodges JS. Comparison of U.S. and Italian experiences with sudden cardiac deaths in young competitive athletes and implications for preparticipation screening strategies. Am J Cardiol 2009;104: 276-80.

171. Halkin A, Steinvil A, Rosso R, Adler A, Rozovski U, Viskin S. Preventing sudden death of athletes with electrocardiographic screening: what is the absolute benefit and how much will it cost? J Am Coll Cardiol 2012;60:2271-6.

172. Fuller CM. Cost effectiveness analysis of screening of high school athletes for risk of sudden cardiac death. Med Sci Sports Exerc 2000;32:887-90.

173. Maron BJ, Thompson PD, Ackerman MJ, Balady G, Berger S, Cohen D et al. Recommendations and considerations related to preparticipation screening for cardiovascular abnormalities in competitive athletes: 2007 update: a scientific statement from the American Heart Association Council on Nutrition, Physical Activity, and Metabolism: endorsed by the American College of Cardiology Foundation. Circulation 2007;115:1643-55.

174. Wheeler MT, Heidenreich PA, Froelicher VF, Hlatky MA, Ashley EA. Costeffectiveness of preparticipation screening for prevention of sudden cardiac death in young athletes. Ann Intern Med 2010;152:276-86.

175. Leslie LK, Cohen JT, Newburger JW, Alexander ME, Wong JB, Sherwin ED et al. Costs and benefits of targeted screening for causes of sudden cardiac death in children and adolescents. Circulation 2012;125:2621-9.

176. Schoenbaum M, Denchev P, Vitiello B, Kaltman JR. Economic evaluation of strategies to reduce sudden cardiac death in young athletes. Pediatrics 2012;130 e380-9.

177. Corrado D, Basso C, Thiene G. Pros and cons of screening for sudden cardiac death in sports. Heart 2013;99:1365-73.

178. Assanelli D, Levaggi R, Carre F, Sharma S, Deligiannis A, Mellwig KP et al. Costeffectiveness of pre-participation screening of athletes with ECG in Europe and Algeria. Intern Emerg Med 2015;10:143-50.

179. Van Camp SP, Bloor CM, Mueller FO, Cantu RV, Olson HG. Nontraumatic sports death in high school and college athletes. Med Sci Sports Exerc 1995;27:641-7.

180. Bohm P, Scharhag J, Meyer T. Data from a nationwide registry on sports-related sudden cardiac deaths in Germany. Eur J Prev Cardiol 2016;23:649-56. 